\title{
HLA class I genotypes customize vaccination strategies in immune simulation to combat COVID-19
}

Shouxiong Huang ${ }^{1,2} *$ Ming Tan ${ }^{3}$

${ }^{1}$ Department of Environmental and Public Health Sciences, University of Cincinnati College of Medicine, Cincinnati, $\mathrm{OH} 45249$, USA

${ }^{2}$ Immunology Graduate Program, Cincinnati Children's Hospital Medical Center, Cincinnati, $\mathrm{OH}$ 45229, USA

3Division of Infectious Diseases, Cincinnati Children's Hospital Medical Center, Cincinnati, OH 45229, USA

Keywords: Binding affinity, human leukocyte antigen class I, CD8 ${ }^{+} \mathrm{T}$ cells, coronavirus disease 2019 (COVID-19), custom vaccination

*Corresponding author. E-mail address: Shouxiong.huang@uc.edu 


\section{Abstract}

Memory $\mathrm{CD}^{+} \mathrm{T}$ cells are associated with a better outcome in Coronavirus Disease 2019 (COVID-19) and recognized as promising vaccine targets against viral infections. This study determined the efficacy of population-dominant and infection-relevant human leukocyte antigens (HLA) class I proteins to present severe acute respiratory syndrome coronavirus 2 (SARS-CoV-2) peptides through calculating binding affinities and simulating $\mathrm{CD} 8{ }^{+} \mathrm{T}$ cell responses. As a result, HLA class I proteins distinguished or shared various viral peptides derived from viruses. HLA class I supertypes clustered viral peptides through recognizing anchor and preferred residues. SARS-CoV-2 peptides overlapped significantly with SARS but minimally with common human coronaviruses. Immune simulation of $\mathrm{CD} 8^{+} \mathrm{T}$ cell activation using predicted SARS-CoV-2 peptide antigens depended on high-affinity peptide binding, anchor residue interaction, and synergistic presentation of HLA class I proteins in individuals. Results demonstrated that multi-epitope vaccination, employing a strong binding affinity, viral adjuvants, and heterozygous HLA class I genes, induced potent immune responses. Therefore, optimal CD8 ${ }^{+} \mathrm{T}$ cell responses can be achieved and customized contingent on HLA class I genotypes in human populations, supporting a precise vaccination strategy to combat COVID19. 


\section{Introduction}

Coronavirus disease 2019 (COVID-19) pandemics has raised more than 55 million cases globally since earlier this year and remains rapid spreading with over a half-million new daily infections worldwide $^{1}$. COVID-19 is caused by the infection of severe acute respiratory syndrome coronavirus 2 (SARS-CoV-2), characterized as pneumonia and lymphopenia, and develops severe respiratory or multiple organ failure in around $5 \%$ of patients ${ }^{2}$. Recent vaccine development mainly focused on the induction of neutralizing antibodies. Although multiple vaccination strategies are under clinical trials ${ }^{3}$, their protection against COVID-19 in large human populations remains unknown. Considering disappointing efficacy or insufficient protection frequently occurred in previous anti-viral vaccine trials using large human populations, alternative approaches to stimulate protective immunity and control infections effectively remain urgently needed.

$\mathrm{CD}^{+} \mathrm{T}$ cells mediate essential immune responses against numerous intracellular viral infections to provide durable T cell memory and protection up to many years after infections, such as in immune defense against SARS ${ }^{4-6}, \mathrm{HIV}^{7,8}$, and influenza virus infections ${ }^{9,10}$. Clinical trials of antiviral vaccines frequently led to disappointing results with weak $\mathrm{CD} 8^{+} \mathrm{T}$ cell responses,8,11, supporting the importance of anti-viral $\mathrm{CD}^{+} \mathrm{T}$ cell responses in different human populations. Indeed, viral-specific CD8 ${ }^{+} \mathrm{T}$ cells are essential for viral clearance and long-term protection against HIV ${ }^{12}$, Ebola ${ }^{13}$, hepatitis B viruses ${ }^{14}$, and cytomegalovirus ${ }^{15}$, despite the critical role of neutralizing antibodies for blocking viral entry to human cells ${ }^{6,16-18}$. In COVID-19, it is likely important to stimulate both neutralizing antibodies and $\mathrm{CD}^{+} \mathrm{T}$ cell responses to achieve full protection, as in viral vaccination trials against $\mathrm{SARS}^{19}$ and influenza virus ${ }^{6,20}$. SARS-CoV-2 infections induce a dramatic reduction of $\mathrm{CD}^{+} \mathrm{T}$ cells associated with severe ${ }^{21,22}$ and elderly patients $^{23}$. Multiple recent reports strongly support that viral-specific and memory CD8 ${ }^{+} \mathrm{T}$ cell responses are associated with milder diseases, younger patients ${ }^{24}$, and convalescent phases of infections ${ }^{25,26}$, although HLA genotype ${ }^{27}$, differentiation kinetics ${ }^{28,29}$, and adjuvant usage ${ }^{30}$ impact the protection of CD8 ${ }^{+} \mathrm{T}$ cells subsets.

Activation of viral-specific CD8 ${ }^{+} \mathrm{T}$ cells and killing of viral-infected cells rely on antigenpresentation mediated by HLA class I proteins together with co-stimulation and cytokine microenvironment ${ }^{31,32}$. A fundamental mechanism is that HLA class I proteins bind specific viral peptides as in a key-to-lock model33,34, which interacts with the T cell receptor of CD8+ T cells 
for activation and viral-infected cell recognition. Similar to the cell entry of SARS-CoV35, SARSCoV-2 is considered to enter lung epithelial cells and blood cells through interacting with cell surface receptors, such as angiotensin-converting enzyme 2 (ACE2), and follow the endocytic and cytoplasmic compartments ${ }^{36}$. Intracellular viral proteins in viral assembling and trafficking processes can be degraded in proteasome or endosome to form antigenic peptide ligands that further bind to HLA class I proteins through a secretory pathway or cross-presentation 37,38 . Currently, computational prediction and experimental identification of peptide antigens for $\mathrm{CD}^{+} \mathrm{T}$ cell activation in COVID-19 are ongoing for a purpose of "identifying vaccine candidates" ${ }^{26,39-41}$. However, the impact of HLA class I genotypes on peptide antigen selection and $\mathrm{CD}^{+} \mathrm{T}$ cell activation remains poorly understood.

To date, the HLA-A locus displays 6291 alleles that encode around 3900 proteins, and HLA-B displays 7562 alleles that encode around 4808 proteins in humans ${ }^{42}$. It is unlikely to fully unveil functional $\mathrm{CD}^{+} \mathrm{T}$ cell responses to unique peptide sets presented by each HLA class I protein. With a wealth of structural knowledge on HLA-peptide-T cell receptor interaction ${ }^{2,43}$, computational prediction and immune simulation as a prototypic artificial intelligent tool become possible and essential to facilitate structural identification and functional simulation of peptide antigen presentation for $\mathrm{CD}^{+} \mathrm{T}$ cell activation. This study applied affinity binding theory to predict SARS-CoV-2 peptides for binding to different HLA class I proteins and simulate the antigen-presentation process for $\mathrm{CD}^{+} \mathrm{T}$ cell activation. Results showed a dramatically different capacity in various HLA class I proteins to bind SARS-CoV-2 peptides. Multiple HLA class I proteins that have been indicated to associate with more severe symptoms or weaker $\mathrm{CD}^{+} \mathrm{T}$ cell responses in $\mathrm{SARS}^{44}$ were demonstrated in this study for binding a less number of SARS-CoV-2 peptides. In contrast, HLA class I proteins that have been suggested protective or potent stimulators of $\mathrm{CD}^{+} \mathrm{T}$ cells in SARS45 were predicted to bind a large number of SARS-CoV-2 peptides. Different HLA class I proteins clustered to a supertype share identical sequences and similar binding patterns of SARS-CoV-2 peptides. Across various coronaviruses, a large number of peptides from SARS viruses and a limited number of peptides from "common cold"-causing human coronaviruses are identical to those of SARS-CoV-2. Further, affinitybased immune simulation provided an optimized combination of peptides dependent on HLA class I genotypes to facilitate customizing precise vaccination strategies for individuals and human populations. 


\section{Results}

Dominant HLA class I proteins sample various numbers of SARS-CoV-2 peptides. To stimulate anti-COVID-19 CD8 ${ }^{+} \mathrm{T}$ cell responses, this study used NetMHCpan program to comprehensively determine the binding affinity of SARS-CoV-2 peptides to ten dominant HLA class I A and class I B proteins, which are frequently detected in the United States (US) ${ }^{46}$. HLA-C alleles were not involved since HLA-C proteins mainly induce immune tolerance ${ }^{47}$. The threshold of sufficient binding affinity was defined using a half-maximal inhibitory concentration $\left(\mathrm{IC}_{5} \mathrm{O}<500 \mathrm{nM}\right)$, in which the maximal concentration of a SARS-CoV-2 peptide is required to competing off the binding of a probe peptide to each HLA class I protein. The spike $(\mathrm{S})$, membrane (M), nucleocapsid (N), and multiple ORF-encoded proteins (Fig. S1A) of the SARS-CoV-2 virus are considered immunogenic and are analyzed in this study because representative peptides of these proteins stimulate $\mathrm{CD} 8^{+} \mathrm{T}$ cells isolated from COVID-19 patients 39,40 . Various numbers of SARS-CoV-2 peptides were predicted with a high binding affinity to ten dominant HLA class I A and B proteins (Fig. S1A). These ten alleles exist in 78.5\% of the US population approximately. Upon the further consideration of the earlier processes in antigen presentation, including proteasome degradation (cutoff $>5$ potential cutting sites by proteasome enzymes) ${ }^{48}$ and peptide transportation through the transporter associated with antigen processing (TAP, cutoff $\mathrm{IC}_{5} \mathrm{O}<1 \mathrm{nM}$ ) 49 , 80\% to $100 \%$ peptide ligands for HLA class I proteins remained significant (Fig. 1A).

As a result, striking variation exists in the numbers of SARS-CoV-2 peptides sampled by different dominant HLA class I proteins. Most of the ten dominant HLA-A and B proteins bound high numbers of peptides derived from noted immunogenic SARS-CoV-2 proteins. $\mathrm{A}^{*}$ 02:01 and $\mathrm{A}^{*}$ 11:01 bound the highest numbers of peptides (Fig. 1), indicating that a large human population expressing at least one of these dominant HLA class I alleles is able to respond to SARS-CoV-2 infection. The capacity of binding a higher number of viral peptides suggests a potential dominancy of viral-induced $\mathrm{CD}^{+} \mathrm{T}$ cell responses. These potent peptide binders can compensate with weak peptide binders and balance the overall ability of antigen presentation for $\mathrm{CD}^{+} \mathrm{T}$ cell activation, if an HLA class I genotype combines both potent and weak peptide binders in the same individual. Apparently, some SARS-CoV-2 proteins provided a lower number of peptides due to their short chain length of proteins, such as N and ORF8-encoded proteins. Thus, to estimate a critical protein source of viral peptides, peptide numbers were further normalized with the chain length of each SARS-CoV-2 protein (Fig. 1B). As a result, 
among different SARS-CoV-2 proteins, fewer percentage of peptides from $\mathrm{N}$ and ORF8-encoded proteins are presented by most HLA-A proteins. Among different HLA class I proteins, HLA-A proteins overall displayed a higher peptide loading capacity than HLA-B proteins. For example, HLA-A*02:01, $A^{*}$ 11:01, and $A^{*} 24: 02$ sampled a higher \% of peptides from multiple viral proteins, except for $\mathrm{N}$ and ORF8-encoded proteins (Fig. 1B). HLA-B*35:01 showed a high peptide loading capacity than other HLA-B proteins. Although the numbers of peptide ligands do not necessarily reflect the protection of peptide antigens in diseases, predicted peptides binding to HLA proteins raise a critical concern that various human populations with various HLA class I genotypes will respond differently in COVID-19 infections.

To consider whether HLA class I sequence heterogeneity selects various numbers of peptide ligands, $\alpha 1$ and $\alpha 2$ ligand-binding domains of ten HLA class I proteins were aligned (Fig. S1B), using a hierarchical clustering approach50. Overall resulted similarity ranged from $75 \%$ to $96 \%$, with the highest similarity between $\mathrm{A}^{*} 03$ :01 and $\mathrm{A}^{*}$ 11:01 (96.15\%) (Fig. S1C). However, the numbers of predicted peptides appear generally unmatched with the degree of similarity between different HLA class I proteins. For example, $A^{*} 02: 01$ shows $92.31 \%$ similarity to $\mathrm{A}^{*}$ 03:01, but they have very different numbers of binding peptides for the same proteins (Fig. 1A). Similar non-concordance occurs between $A^{*} 01: 01$ and $A^{*} 11: 01$ with $93.96 \%$ sequence similarity and between $A^{*} 03: 01$ and $A^{*} 11: 01$ with the highest sequence similarity of $96.15 \%$. These results suggest a need to exam the similarity of peptide-binding domains among HLA class I proteins.

Dominant HLA class I proteins share limited numbers of SARS-CoV-2 peptides. To determine the conservation of viral peptides for $\mathrm{CD}^{+} \mathrm{T}$ cell activation in different human populations, we applied Venn diagrams to show the numbers of predicted identical peptides between dominant HLA-A proteins (Fig. 2A) and HLA-B proteins (Fig. 2B). We used the spike and ORF1ab genes, which encode longer protein sequences than others, to provide a higher chance of overlapped peptide sampling by different HLA class I proteins. As a result, identical peptides are infrequently shared by many HLA class I proteins despite high sequence similarity (>90\%) between HLA-A or HLA-B proteins (Fig. S1C). For some HLA proteins, vast numbers of shared peptides were observed, for example, between $\mathrm{A}^{*} 03: 01$ and $\mathrm{A}^{*}$ 11:01 with an extremely high sequence similarity of $96.15 \%$, across $B^{*} 40: 01$ and $B^{*} 44: 03$ with a relatively low similarity of $89.56 \%$, or between $\mathrm{B}^{*} 35: 01$ and $\mathrm{B}^{*} \mathrm{07:02}$ with a lower sequence similarity of $88.46 \%$. In particular, almost $80 \%$ or $85 \%$ of peptides bound to $\mathrm{A}^{*} \mathrm{0} 3: 01$ also bind to $\mathrm{A}^{*} 11: 01$. However, 
HLA sequence similarity is overall discordant to the numbers of shared peptides. $\mathrm{B}^{*} \mathrm{08:01}$ and $B^{*}$ 07:02 (93.41\% sequence similarity) shared much fewer numbers of peptides, and B*08:01 and $B^{*} 40: 01$ (92.31\% sequence similarity) shared no peptide from the S protein or only 1 out of 340 peptides from the ORF1ab-encoded proteins. Therefore, an overall high sequence similarity does not suggest a high number of peptide overlap. As in the sequence alignment (Fig. S1B), potential determinants for the numbers of shared peptides likely originate from hot spots with limited numbers of residues in ligand-binding grooves. The similarity of these key residues and pocket structures assigns HLA class I proteins to multiple supertypes ${ }^{51,52}$. Indeed, high numbers of identical SARS-CoV-2 peptides were shared between HLA class I proteins of the same supertypes, such as supertypes Ao3 ( $\mathrm{A}^{*} 03: 01$ and $\mathrm{A}^{*}$ 11:01), Bo7 ( $\mathrm{B}^{*} 35: 01$ and $\left.\mathrm{B}^{*} 07: 02\right)$, and B40 (B*40:01 and B*44:03) (Fig. 2).

Further, peptide-binding motifs of HLA class I proteins interact with anchor and preferred residues of peptide ligands to achieve high binding affinity (Fig. 3). The unique or shared anchor residues validates the binding of predicted SARS-CoV-2 peptides to specific HLA class I proteins or to HLA class I supertypes, respectively. Thus, the GibbsCluster program53,54 was applied to determine the residue patterns of predicted SARS-CoV-2 peptides. As a result, SARS-CoV-2 peptides showed conserved residues at positions 2 or 3 and 9, matching the reported anchor residues or preferred residues with corresponding HLA class I proteins (Fig. 3). HLA class I proteins assigned to the same supertypes shared similar anchor and preferred residues, as for supertypes B40 (B*40:01 and B*44:03), and to a less degree for Bo7 ( ${ }^{*} 35: 01$ and B*07:02)55-60. For $\mathrm{A}^{*}$ 03:01 with undefined anchor and preferred residues, this study showed a similar pattern of peptide binding to $A^{*} 03: 01$ as to $A^{*} 11: 01$, which display a sequence similarity of $96.15 \%$ (Fig. 1C) and belong to Ao3 supertype ${ }^{52}$. Therefore, predicted SARS-CoV-2 peptides form various patterns of anchor and preferred residues, which are shared within an HLA class I supertype (Fig. 3), as a unique conserved mechanism to implement HLA genotype-customized CD8+ T cell activation in various human populations.

\section{HLA class I proteins with weak peptide binding capacity incline to associate with} severe infections. Multiple HLA class I proteins have been reported to associate with more severe diseases of viral infections. In SARS infections, B*46:01 was associated with severe cases in a study using a small sample size 44 and the association of $\mathrm{B}^{* 07: 03}$ with severe SARS could not pass multiple tests ${ }^{61}$. In contrast, peptide binding to the HLA class I proteins of Ao3 supertype, including $\mathrm{A}^{*} 03: 01$ and $\mathrm{A}^{*}$ 11:01, was considered as potent stimulators for $\mathrm{CD}^{+} \mathrm{T}$ cells against 
SARS45, and the potential association of $\mathrm{B}^{*} 15: 02$ with resistance to SARS is not statistically significant in multiple tests ${ }^{61}$. Since individuals usually express two to four heterozygous or homozygous HLA class I A and B paralogous proteins, the potential association of one protein with severe diseases is likely balanced or interfered by other heterozygous HLA class I proteins in human population studies. The numbers of predicted peptide ligands in our study are supportive of a potential association of these HLA class I proteins with severity or protection of infections. For potent peptide binders potentially associated with resistance or protection, $\mathrm{A}^{*}$ 02:01, $\mathrm{A}^{*}$ 11:01, and $\mathrm{B}^{*}$ 15:03 sampled a large number of peptides (Fig. 4A). These results are consistent with a common stimulator of $\mathrm{A}^{*} 11: 01$ for anti-SARS CD8 ${ }^{+} \mathrm{T}$ cell responses ${ }^{45}$, a potential association of $\mathrm{B}^{*} 15: 02$ with the resistance to $\mathrm{SARS}^{61}$, and needs to further understand the effector phenotypes ${ }^{27}$ and kinetics ${ }^{29}$ of $\mathrm{A}^{*}$ O2-mediated CD8 ${ }^{+} \mathrm{T}$ cell responses in COVID-19. Belonging to the same supertypes (Fig. 4) and share high sequence homology (Fig. S2), A*02:02 sampled a high number of peptides as for $\mathrm{A}^{*} \mathrm{02:01}$ (A02 supertype), and the similar was observed for $B^{*} 15: 01, B^{*} 15: 02$, and $B^{*} 15: 03$, although a controversy remains in their assignment to one supertype or two supertypes ${ }^{2,62}$. Interestingly, SARS-CoV-2 peptides were predicted to be overlapped between $A^{*} 02$ and $B^{*} 15$ proteins or between $A^{*} 11$ and $B^{*} 15$ proteins (Fig. $4 \mathrm{~B}$ ), providing an additional possibility of identical peptides unusually shared by paralogous HLA class I proteins. On the other side, relative weak peptide binders $B^{*} 07: 03$ and $B^{*} 46: 01$ in this study were suggested potentially associated with severe SARS in some studies ${ }^{61}$ or with a small sample size44. B*07:02 and B*07:03 alleles (B07 supertype) sampled relatively fewer numbers of peptides in comparison to potent peptide binders such as $\mathrm{A}^{*} \mathrm{02}: 02$ or $\mathrm{B}^{*} 15: 03$, consistent with its difficulty to pass multiple tests in severe SARS ${ }^{61}$ if co-expressed with strong binders. Surprisingly, $B^{*} 46: 01$ is predicted almost not binding to peptides from SARS-CoV-2 (Fig. 4A), suggesting a potential weak $\mathrm{CD}^{+} \mathrm{T}$ cell response against COVID-19 and consistent to its association with severe SARS infection $44 . A^{*} 25: 01$ binds a very limited number of SARS-CoV-2 peptides at low binding affinity, indicating a potential concern of weak responses in patients with an $\mathrm{A}^{*}$ 25:01 allele. These results allow us to predict and prepare to overcome weak peptide presenters in fighting human COVID-19 infections.

\section{Overlapped HCoV and SARS-CoV-2 peptides suggests cross-reactive $\mathrm{CD8}^{+} \mathbf{T}$ cells.} Recent tests demonstrated that non-infected healthy donors contain $\mathrm{CD}^{+} \mathrm{T}$ cells responding to SARS-CoV-2 peptides 39,40 . Therefore, one can hypothesize that "common cold" coronavirus infection in humans can generate cross-reactive $\mathrm{CD}^{+} \mathrm{T}$ cells against SARS-CoV-2 infections. Moreover, cross-reactivity is more prominent and much stronger between SARS and SARS-CoV- 
$2^{40}$. Our predicted numbers of overlapped peptides can overall explain this cross-reactivity. SARS and SARS-CoV-2 share various sequence similarity, such as 77.54\% for S protein (Fig. S3) and $95.37 \%$ for ORF1b-encoded proteins (Fig. S4), contributing to the overlap of 69 peptides from spike protein and 438 peptides from ORF1b-encoded proteins between SARS with SARSCoV-2 (Fig. 5). Since CD8 ${ }^{+} \mathrm{T}$ cells exposed to "common cold" coronaviruses are potentially reactive to SARS-CoV-2 peptides, the prediction of human coronavirus peptides contributing to this cross-reactivity will provide bases for cross-virus protection. However, $\mathrm{HCoV}-\mathrm{OC} 43$ and SARS-CoV-2 share much lower sequence similarity at 33.06\% for S protein (Fig. S3) and $62.32 \%$ for ORF1b-encoded proteins, contributing to 44 shared peptides (5\%) from ORF1b-encoded proteins but nearly zero shared peptide from $\mathrm{N}, \mathrm{S}$, and ORF1a-encoded proteins between $\mathrm{HCoV}$ OC43 and SARS-CoV-2 (Fig. 5). This limited number of cross-reactive peptides from $\mathrm{HCoV}$ is valuable for further functional investigations for cross protection.

Heterozygous HLA class I genotypes with high affinity peptides enhance $\mathrm{CD8}^{+} \mathbf{T}$ cell responses in immune simulation. To predict whether high peptide binding affinity, peptide numbers, and HLA class I heterozygosity enhance $\mathrm{CD} 8^{+} \mathrm{T}$ cell responses, we use an agent-based simulator, C-ImmSim, to simulate antigen presentation for $\mathrm{CD}^{+}{ }^{+} \mathrm{T}$ cell activation ${ }^{63}$. C-ImmSim mainly incorporated the impact of HLA-peptide binding, innate signaling, memory responses, and adjuvant effect in antigen presentation and $\mathrm{CD} 8^{+} \mathrm{T}$ cell activation, particularly differentiating the effect of heterozygosity or homozygosity of HLA genes ${ }^{63}$. First, we compared $\mathrm{CD}^{+}{ }^{+} \mathrm{T}$ cell responses to one versus ten peptides with a high binding affinity (IC50 $<16 \mathrm{nM}$ ) without involving innate or adjuvant factors. Results showed that one peptide is insufficient, but a combination of ten peptides, stimulated $\mathrm{CD}^{+} \mathrm{T}$ cell responses that became saturated in around two weeks (Fig. 6A). In this simulation, multi-epitopes stimulated more robust $\mathrm{CD}^{+} \mathrm{T}$ cell responses than one epitope, possibly due to very strong total binding avidity. Second, to more precisely test the avidity hypothesis, five peptides with the highest IC50 were compared with the other five peptides with the lowest $\mathrm{IC}_{50} \mathrm{O}$ as loaded to the same $\mathrm{A}^{*} \mathrm{O2}$ :01 protein. Results further supported the conclusion that strong $\mathrm{CD}^{+} \mathrm{T}$ cell responses are more likely to be activated by multiple peptides with a high binding affinity (low IC50) to HLA class I proteins (Fig. 6B). Further, to determine whether heterozygous HLA-A or HLA-B proteins show an advantage to enhance $\mathrm{CD}^{+} \mathrm{T}$ cell responses than homozygous $\mathrm{A}^{*} \mathrm{02:01}$ or $\mathrm{B}^{*} 44: 02$ alone, results showed peptides with similar or lower binding affinity stimulated stronger $\mathrm{CD} 8^{+} \mathrm{T}$ cell responses by heterozygous HLA-A or B proteins. Moreover, paralogous HLA-A and B proteins showed similar strength for $\mathrm{CD} 8{ }^{+} \mathrm{T}$ cell activation to heterozygous HLA class I A or B proteins 
(Fig. 6C). Therefore, high peptide binding affinity, HLA gene heterogeneity, and paralogous HLA-A and B proteins provide advantages to stimulate a robust $\mathrm{CD} 8^{+} \mathrm{T}$ cell response.

\section{Heterozygous HLA class I genotypes with viral adjuvants rescue $\mathrm{CD8}^{+} \mathrm{T}$ cell} responses in immune simulation to weak peptide binders. Although $B * 46: 01$ and $\mathrm{B}^{*}$ 07:02 are potentially relevant to severe SARS44,61 and shown as weak peptide binders (Fig. 4), we speculated that $\mathrm{CD} 8^{+} \mathrm{T}$ cell responses in the individuals expressing one of these alleles can still be strongly stimulated by optimizing peptide immunization utilizing HLA class I heterogeneity and viral adjuvant. Strikingly, results showed that only three predicted peptides from multiple SARS-CoV-2 proteins bind to $\mathrm{B}^{*} 46: 01$ at a sufficient affinity (IC50 $<500 \mathrm{nM}$ ) in comparison to hundreds or thousands of peptides sampled by other HLA class I proteins (Fig. 4A). One can imagine that an individual with a $B^{*} 46: 01$ allele will be challenging for inducing $\mathrm{CD}^{+} \mathrm{T}$ cell activation, as this likely explains why $\mathrm{B}^{*} 46: 01$ allele is associated with severe SARS in a study using a small sample size44. Thus, it is critical to determine whether other HLA-A or B alleles in the same individuals facilitate overcoming this weakness. For example, HLA-A ${ }^{*}$ 02:07 and HLA-B* $46: 01$ haplotype is present in around 3.34\% of Asian pacific population ${ }^{64}$. Whether the peptides presented by HLA-A ${ }^{*} 02: 07$ can rescue or enhance $\mathrm{CD}^{+} \mathrm{T}$ cell responses mediated by the weak peptide binder $\mathrm{B}^{*} 46: 01$ is critical for inducing anti-COVID-19 immunity in this human population. By applying one additional peptide that is presented by a paralogous $\mathrm{A}^{*}$ 02:07 or by a heterozygous $\mathrm{B}^{*} 07: 02$ protein, results supported both strategies of antigen presentation overcame the disadvantage of $\mathrm{B}^{*} 46: 01$ protein for $\mathrm{CD}^{+} \mathrm{T}$ cell activation (Fig. 4A). Furthermore, the application of adjuvants such as an attenuated viral strain will help provide a cytokine environment mediated by innate cells and $\mathrm{CD}^{+} \mathrm{T}$ cells to facilitate the activation of peptide-specific $\mathrm{CD}^{+} \mathrm{T}$ cells (Fig. $7 \mathrm{~B}$ and $7 \mathrm{C}$ ). In this immune simulation, the viral adjuvant enhanced the proliferation of peptide-specific memory $\mathrm{CD} 8^{+} \mathrm{T}$ cells and balanced the production of IFN $\gamma$ and inhibitory cytokines with extended kinetics. Therefore, a precise vaccination strategy considering potent peptide binders, heterozygous and paralogous advantages of HLA class I alleles, multi-epitopes of peptide antigens, and a viral adjuvant will induce strong anti-viral $\mathrm{CD}^{+} \mathrm{T}$ cell responses as tailored to individual and populational HLA class I genotypes. 


\section{Discussion}

More than two, three, and ten thousand HLA-A and B alleles have been detected in Asian, African, and European Americans, respectively 64 . The same viral proteins are expected to induce divergent $\mathrm{CD} 8{ }^{+} \mathrm{T}$ cell responses in different individuals or populations, demanding a precise determination of efficacious peptide antigens presented by individual-specific HLA class I proteins. Remarkably extensive efforts are needed to structurally profile and functionally validate viral peptide pools for various HLA class I genotypes in ethnically diverse human populations. Computational prediction of these tens of thousands of peptides in terms of their binding affinity to each HLA class I proteins from tens of hundreds of HLA class I genotypes provides highly feasible alternatives to predict candidate peptides. Further, $\mathrm{CD}^{+} \mathrm{T}$ cell activation using these large numbers of combinations of predicted peptides and HLA class I genotypes can only be possibly performed using immune simulation to provide limited candidate strategies for further experimental and clinical tests. This study performed a computational prediction of peptide ligands and an immune simulation of $\mathrm{CD}^{+} \mathrm{T}$ cell activation using representative potent and weak peptide binders, including ten dominant HLA class I alleles and potentially SARS-associated HLA class I alleles. Results demonstrated that multiepitope high-affinity peptides, tailored to heterozygous and paralogous HLA class I genotypes combined with a viral adjuvant, elicits strong $\mathrm{CD}^{+} \mathrm{T}$ cell responses, memory $\mathrm{CD}^{+} \mathrm{T}$ cell differentiation, and sustainable cytokine production.

Regardless of the degree of sequence diversity (Fig. S1C), key residues in peptide-binding motifs define the supertypes of HLA class I proteins ${ }^{51,52}$. HLA class I proteins of the same supertypes share peptide antigens and peptide-binding patterns, suggesting the conservation of antiCOVID-19 antigenic peptides in human populations. Briefly, large numbers of identical peptides overlapped between $A^{*} 03: 01$ and $A^{*}$ 11:01 (Ao3 supertype), $B^{*} 44: 03$ and $B^{*} 40: 01$ (B40) (Fig. 2), $\mathrm{A}^{*}$ 02:01 and $\mathrm{A}^{*}$ 02:02 (A02), $\mathrm{B}^{*} 07: 02$ and $\mathrm{B}^{*} 07: 03$ (Bo7), and $\mathrm{B}^{*} 15: 01$ and $\mathrm{B}^{*}$ 15:03 (B27) (Fig. 4). In these HLA class I supertypes, $A^{*} 02: 01$ and $A^{*} 02: 02$ shared more than $50 \%$ of identical peptides, and three $\mathrm{B}^{*} 15$ proteins shared large numbers of identical peptides (Fig. 4). Overlaps between paralogous HLA-A*02 and $\mathrm{B}^{*} 15$ proteins also occurred at a much lower degree, implicating the presence of degenerate epitopes for $\mathrm{CD}^{+} \mathrm{T}$ cells ${ }^{65}$. Therefore, by defining HLA class I genotypes in targeted populations, similar or identical peptide antigens shared by HLA class I supertypes can be applied to vaccine design for those populations expressing HLA class I genes of the same supertypes. 
Peptides with high affinity and avidity stimulate robust $\mathrm{CD}^{+} \mathrm{T}$ cell responses and aid weak peptide binder for $\mathrm{T}$ cell activation, as demonstrated by the immune simulation of antigen presentation in this study. One of our selected peptide, "FIAGLIAIV", presented by HLA$\mathrm{A}^{*}$ 02:01 has been validated for the activation of T cell from SARS patients ${ }^{66}$. Evidence was provided with insufficiency for stimulating $\mathrm{CD}^{+} \mathrm{T}$ cell responses using a single peptide (Fig. 6A) or five low-affinity peptides (Fig. 6B) in a vaccination setting in comparison to strong responses with ten peptides or five high-affinity peptides, respectively. Therefore, a high total peptide binding affinity (or avidity) is crucial for $\mathrm{CD}^{+} \mathrm{T}$ cell activation in vaccination.

Heterozygosity of HLA alleles is considered as a result of balancing selection to shape exceptional polymorphism of HLA proteins and increase host immunocompetence against highly diverse pathogens ${ }^{67,68}$. In this study, HLA class I heterozygosity was demonstrated to provide an advantage in presenting additional high-affinity peptides complementarily (Figs. 6 and 7). This heterozygous advantage allows the design of optimal peptide vaccination to achieve pronounced $\mathrm{CD}^{+} \mathrm{T}$ cell responses. For example, HLA-B* $46: 01$ that was predicted to bind to two low-affinity peptides from ORF1ab-encoded proteins, in comparison to $\mathrm{B}^{*} 15: 03$ and $\mathrm{B}^{*} \mathrm{07:02}$ predicted to bind 1538 and 158 peptides derived from the same SARS-CoV-2 proteins (Fig. 4), likely supports the theory of heterozygous advantage ${ }^{68}$, if these HLA class I alleles express in an individual. This study provided an example of immune simulation that $\mathrm{CD}^{+} \mathrm{T}$ cell responses in an individual expressing a weak peptide binder HLA-B*46:01 protein can be enhanced and rescued by the heterozygous expression of $\mathrm{B}^{*} 07: 02$ protein, which provided complementary capacity in peptide presentation to enhance $\mathrm{CD}^{+} \mathrm{T}$ cell activation (Fig. $7 \mathrm{~A}$ ). This heterozygous advantage has also been clinically demonstrated in individuals with heterozygous HLA class I loci A and B for protection against human T-lymphotropic virus type-1 (HTLV-1) ${ }^{68,69}$. Thus, HLA heterozygosity is an advantageous factor to be critically examined, particularly in the populations or individuals with weak $\mathrm{CD} 8+\mathrm{T}$ cell responses to the virus- or protein-based vaccines, which can be rescued by providing a custom design of peptide antigens for the presentation of heterozygous HLA class I proteins.

Dual antigen presentation mediated by paralogous HLA-A and B proteins, as existing in all HLA class I haplotypes, further enhances $\mathrm{CD}^{+} \mathrm{T}$ cell responses. This dual antigen presentation elicits more robust $\mathrm{CD}^{+} \mathrm{T}$ cell responses than the antigen presentation mediated by a single HLA-A or B protein for a similar number of peptides (Fig. 6C). Using the weak peptide binder $B^{*} 46: 01$ as 
an example, peptides presented by HLA-A proteins can be applied to rescue the weak $\mathrm{CD} 8^{+} \mathrm{T}$ cell responses mediated by HLA-B*46:01 protein. For example, in clinical trials of anti-viral vaccination, 3.34\% of the Asian Pacific population co-expressing HLA-A*02:07 and the weak peptide binder HLA-B* 46:0164 can be considered to have additional peptide-based vaccination to elicit optimal CD8 ${ }^{+} \mathrm{T}$ cell responses, as customized to this specific HLA class I genotype (Fig. 7). Moreover, viral co-vaccination or adjuvant can be applied to boost more robust or stabilized $\mathrm{CD}^{+} \mathrm{T}$ cell responses for the haplotype containing B*46:01 in human populations, together with antigen presentation mediated by either heterozygous or paralogous HLA class I A and B proteins (Fig. 7).

In summary, precision vaccination strategies targeting $\mathrm{CD}^{+} \mathrm{T}$ cells can be customized on HLA class I genotypes and peptide affinity for human populations to circumvent weak peptide binders in T cell activation. Dependent on HLA class I genotypes from an individual or a population, high-affinity peptides can be matched to heterozygous or paralogous HLA class I A and $B$ alleles together with adjuvant as customized vaccination strategies. This key-to-lock model33,34 explains why vaccine efficacy results are usually very different between animal experiments and human trials, because antigen-presenting molecules and peptide antigens for $\mathrm{T}$ cell activation are entirely different between animals, including primates, and humans. However, clinical trials to determine the efficacy of a vaccine is time- and labor-consuming, and economically challenging, due to the requirement for double-blind design and the observation of the protection under natural infection conditions in a long-term process. Computational simulation of antigen presentation, using this study as an example, provides valuable candidate strategies for HLA genotype-customized vaccine design using known viral protein sequences. It is reasonable to speculate that the antigen presentation tailored to HLA genotypes of patients is advantageous to overcome weak $\mathrm{CD}^{+} \mathrm{T}$ cell reactivity and facilitate achieving a high efficacy of protection in anti-SARS-CoV-2 vaccine trials clinically.

\section{Materials and Methods}

1. Protein sequences were accessed from GenBank with accession numbers of QHU79204.1 (spike or S protein), QLI50116.1 (membrane or M protein), QHU79211.1 (nucleocapsid or N protein), QJQ39966 (open reading frame 1ab or ORF1ab), QJQ27841 (ORF1a), QJQ39969 (ORF3a), QJQ27861 (ORF8), Protein sequences of human beta coronavirus OC43 isolate include AGT51680.1 (S protein), AIX10719.1 (N protein), 
YP_009555238.1 (ORF1ab), and QBP84755.1 (ORF1a). Protein sequences of SARS include AAP51227.1 (S protein), AAP51234.1 (N protein), QJQ39966 (ORF1ab), QJQ27841 (ORF1a). ORF1b fragment is defined by the non-overlapped portions of amino acid sequences between ORF1ab and ORF1a. HLA class I protein sequences for peptide binding prediction were accessed from the website of The European Molecular Biology Laboratory (EMBL)-European Bioinformatics Institute (EBI) (https://www.ebi.ac.uk/ipd/imgt/hla/allele.html).

2. The alignment of protein sequences was performed using a hierarchical clustering approach (http://multalin.toulouse.inra.fr/multalin/)50. Neutral consensus residues are labeled in red, low consensus residues are labeled in blue, and high consensus portions are labeled in grey as a background. Upon sequence alignment, percentages of identical sequences are calculated and listed.

3. Affinity-based prediction of peptide ligands for HLA class I proteins. The model NetMHCpan was chosen because this model has been widely used to predict the binding affinity of peptide sequences with 8-11 residues to various HLA class I proteins. NetMHCpan calculates a 50\% maximal inhibitory concentration (IC50) based on the maximal concentration of test peptides to compete off a probe peptide for binding to the same HLA class I protein ${ }^{46}$. NetMHCpan prediction has been performed accurately in recent antigen peptide prediction to integrate mass spectrometry-eluted ligands and binding affinity of peptides ${ }^{70,71}$. NetMHCpan is also available for all MHC alleles to analyze dominant and disease-associated HLA class I alleles. This study used the NetMHCpan 4.0 program, which is a default method in the website of Immune Epitope Database (IEDB.org) for predicting peptide ligand binding to HLA class I proteins. This prediction was not limited to the most typical length of peptide sequence ( 9 amino acids) but expanded to cover a possible length of 8 to 11 residues.

4. Proteasome degradation mainly considers the degradation mediated by immune proteasomes, which are induced by Interferon $\gamma$ to generate antigenic peptides. Immune proteasomes degrade SARS-CoV-2 proteins to polypeptides, which have a high potential to bind to MHC Class I molecules. The prediction was based on in vitro proteasomal digestion of enolase and casein proteins as described 48 to determine the numbers of potential cutting sites at the C-termini induced by various proteasome proteases. In this 
prediction, we used the Netchop v3.0 "C-term" model to remove any peptides that were not predicted via proteasomal cleavage of the peptide's $\mathrm{C}$ terminus ${ }^{72,73}$. A conserved threshold of five was used to select peptide ligands with five or more cutting sites in proteasome degradation to generate peptides for further canonical MHC class I antigen processing.

5. Transportation by TAP transporters. Upon proteasome digestion, cytosol peptides are required to be transported by peptide transporters across the endoplasmic reticulum membrane to be loaded to the heavy chain of HLA class I proteins. It has been shown that a high affinity of TAP transporter binding to a peptide translates into high transport rates ${ }^{49}$. Notably, both proteasome and TAP predictions were developed using experimental data for HLA proteins and were highly suitable for predicting viral peptide ligands for HLA class I proteins. Interestingly, TAP ligands with high binding affinity have an increased chance of being cleaved by the proteasome, and TAP specificity has evolved to fit the digestion specificity of proteasomes 73 . Thus, the prediction of TAP transporter binding to peptides links the processes of proteasome digestion and peptide loading to HLA class I proteins. A TAP score estimates - $\log \left(\mathrm{IC}_{5} \mathrm{O}\right)$ values (log=base 10) of peptides for binding to TAP. The cutoff of $\mathrm{IC}_{5} \mathrm{O}$ at $1 \mathrm{nM}$ was used to remove peptides with higher IC50 or lower TAP binding affinity49.

6. Alignment of peptide residues. We align short peptides using the computational program Gibbs (http://www.cbs.dtu.dk/services/GibbsCluster/), which performs two essential tasks simultaneously, alignment and clustering54. We used Gibbs to deconvolute binding motifs of SARS-CoV-2 peptide datasets. GibbsCluster simultaneously clusters and aligns peptide data as a powerful tool for unsupervised motif discovery. Results return optimal clusters with characterized sequence motifs for each cluster based on multiple parameters, including adjustable penalties for small clusters, adjustable penalties for overlapping groups, and a trash cluster to remove outliers. The results of cluster peptides are shown with the frequency of specific amino acids. The sequence motifs derived from the best solution are displayed in the form of sequence logos generated with Seq2Logo. Colors highlight different categories of amino acids, including acidic (red), basic (blue), uncharged (green), and other residues (black). The preidentified anchor and preferred residues of previously defined peptide ligands for HLA class I proteins were obtained from 
(http://www.syfpeithi.de/bin/MHCServer.dll/FindYourMotif.htm) to compare with the enriched clusters of predicted SARS-CoV-2 peptides.

7. Venn diagram was constructed using the website (http://bioinformatics.psb.ugent.be/cgi-bin/liste/Venn/calculate_venn.htpl) for fourway and five-way Venn diagrams. Sequentially identical or overlapped peptide sequences are defined as identical or overlapped peptides.

8. Immunity simulation was performed using C-ImmSim program (http://150.146.2.1/C-IMMSIM/index.php?page $=0$ ) ${ }^{63}$. This prediction program implements a Celada-Seiden model as a logical description of the mechanisms making up humoral and cellular immune responses to a genetic antigen by incorporating known principal factors. These core factors include MHC restriction, clonal selection by antigen affinity, antigen presentation in cytosolic or endocytic pathways, cell-cell interactions, $\mathrm{T}$ cell differentiation, and $\mathrm{T}$ cell memory for $\mathrm{T}$ cell responses. Simultaneously, C-ImmSim considers the helper function of $\mathrm{CD} 4^{+} \mathrm{T}$ cell to antibody production and $\mathrm{CD} 8^{+} \mathrm{T}$ cell responses. C-ImmSim has been used to predict immune response in HIV infections, which have been validated with published clinical trials involving antiretroviral therapies 74 . This study utilized C-ImmSim to predict whether peptide binding affinity, peptide numbers, and combined HLA class I alleles impacted $\mathrm{CD}^{+} \mathrm{T}$ cell response to SARS-CoV-2 peptides. Input factors included peptide sequences, HLA class I alleles, incorporation of a viral vaccination as an adjuvant for this study. Results are shown with the curve of $\mathrm{CD}^{+} \mathrm{T}$ cell proliferation and cytokine production to predict potential effector $\mathrm{T}$ cell responses and memory $\mathrm{T}$ cell differentiation stimulated by HLA class Irestricted peptides.

\section{Acknowledgement}

Thanks to the American Lung Association for funding support (IA-629987).

\section{Author Contributions}

S.H. developed research workflow, analyzed data, and wrote the manuscript. M.T. provided inputs on viral vaccination and manuscript writing. 
Figure legends:

Fig. 1. Dominant HLA class I proteins sampled various numbers of SARS-CoV-2 peptides. Peptide ligands were predicted based on binding affinity to HLA class I proteins, digestion sites for proteasome enzymes, and binding affinity to TAP transporter. The numbers of predicted peptide ligands from immunogenic SARS-CoV-2 proteins are listed for ten dominant HLA class I proteins (A). \% of the numbers of predicted peptides over the amino acid numbers of different SARS-CoV-2 proteins are plotted to reflect the relative frequencies of HLA class I peptide ligands (B).

Fig. 2. Dominant HLA class I proteins shared limited numbers of SARS-CoV-2 peptides. Numbers of predicted SARS-CoV-2 peptide ligands are shown for HLA-A proteins (A) and HLA-B proteins (B).

Fig. 3. Enriched residues in predicted SARS-CoV-2 peptide ligands. Anchor and preferred residues for each HLA class I proteins are compared with enriched residues from predicted SARS-CoV-2 peptide ligands. Peptide ligands from the spike and ORF1ab-encoded proteins are shown for HLA-A proteins (A) and HLA-B proteins (B).

Fig. 4. HLA class I proteins with weak peptide binding capacity inclined to associate with severe infections. Peptide ligands from immunogenic SARS-CoV-2 proteins were predicted for HLA class I proteins that were indicated relevant or irrelevant to the severity or protection of SARS. The numbers of predicted peptide ligands are listed for different dominant HLA class I proteins (A). Venn diagrams show the numbers of predicted peptide ligands from the spike and ORF1ab-encoded proteins for HLA class I proteins (B). White and yellow numbers are for overlaps by two and multiple HLA class I proteins, respectively.

Fig. 5. Overlapped peptides between SARS-CoV-2 and other coronaviruses. A. Venn diagrams show the numbers of identical predicted peptides shared between SARS-CoV-2 and other coronaviruses for HLA class I proteins. Large numbers show the overlaps between SARSCoV-2 and other coronaviruses (white). Numbers of identical peptides loaded to both HLA-A and HLA-B proteins are shown in yellow. B. \% of identical peptides between SARS-CoV-2 and other coronaviruses are listed. Bold \% is for overlaps between SARS-CoV-2 and HCoV-OC43. 
Fig. 6. Heterozygous HLA class I genotypes with high affinity peptides enhance $\mathrm{CD8}^{+} \mathrm{T}$ cell responses in immune simulation. Simulations of antigen presentation are compared with one and ten peptide ligands for $\mathrm{A}^{*}$ 02:01 protein (A). Simulations of antigen presentation are compared between the high and low binding affinity of five peptide ligands for $\mathrm{A}^{*}$ 02:01 protein (B). Simulations of antigen presentation are compared between homozygous (i) and heterozygous (ii) HLA-A proteins, homozygous (iii) and heterozygous (iv) HLA-B proteins, and with paralogous HLA-A and B proteins (vi) (C).

Fig. 7. Heterozygous HLA class I genotypes with viral adjuvants rescue $\mathrm{CD}^{+} \mathrm{T}^{+}$cell responses in immune simulation to weak peptide binders. Simulations of antigen presentation used $B^{*}$ 46:01 only, mixed HLA-A and B*46:01, and heterozygous HLA-B alleles with $B^{*} 46: 01$ (A). Simulations of antigen presentation used viral adjuvant and peptide ligands for both HLA-A and B*46:01 proteins (B). Simulations of antigen presentation used viral adjuvant and peptide ligands for heterozygous HLA-B proteins with $B^{*} 46: 01$ (C). 


\section{References}

1 Dong, E., Du, H. \& Gardner, L. An interactive web-based dashboard to track COVID-19 in real time. Lancet Infect Dis 2o, 533-534, doi:10.1016/S1473-3099(20)30120-1 (2020).

2 Cao, X. COVID-19: immunopathology and its implications for therapy. Nat Rev Immunol 2o, 269270, doi:10.1038/s41577-020-0308-3 (2020).

3 Thanh Le, T. et al. The COVID-19 vaccine development landscape. Nat Rev Drug Discov 19, 305-306, doi:10.1038/d41573-020-00073-5 (2020).

4 Channappanavar, R., Zhao, J. \& Perlman, S. T cell-mediated immune response to respiratory coronaviruses. Immunologic research 59, 118-128, doi:10.1007/s12026-014-8534-z (2014).

$5 \mathrm{Ng}$, O. W. et al. Memory T cell responses targeting the SARS coronavirus persist up to 11 years postinfection. Vaccine 34, 2008-2014, doi:10.1016/j.vaccine.2016.02.063 (2016).

6 Huang, S., He, Q. \& Zhou, L. T cell responses in respiratory viral infections and chronic obstructive pulmonary disease. Chin Med $J$ (Engl) 133, In press (2020).

7 Collins, D. R., Gaiha, G. D. \& Walker, B. D. CD8(+) T cells in HIV control, cure and prevention. Nat Rev Immunol 20, 471-482, doi:10.1038/s41577-020-0274-9 (2020).

8 Watkins, D. I. The hope for an HIV vaccine based on induction of CD8+ T lymphocytes--a review. Mem Inst Oswaldo Cruz 103, 119-129, doi:10.1590/s0074-02762008000200001 (2008).

9 Mullbacher, A., Lobigs, M., Alsharifi, M. \& Regner, M. Cytotoxic T-cell immunity as a target for influenza vaccines. Lancet Infect Dis 6, 255-256, doi:10.1016/S1473-3099(06)70443-1 (2006).

10 Wang, Z. F. et al. Recovery from severe $\mathrm{H}_{7} \mathrm{~N}$ 9 disease is associated with diverse response mechanisms dominated by CD8(+) T cells. Nat Commun 6, doi:ARTN 6833

10.1038/ncomms7833 (2015).

11 Tameris, M. D. et al. Safety and efficacy of MVA85A, a new tuberculosis vaccine, in infants previously vaccinated with BCG: a randomised, placebo-controlled phase $2 \mathrm{~b}$ trial. Lancet 381, 1021-1028, doi:10.1016/So140-6736(13)60177-4 (2013).

12 Fong, Y. Y. et al. Modification of the Association Between T-Cell Immune Responses and Human Immunodeficiency Virus Type 1 Infection Risk by Vaccine-Induced Antibody Responses in the HVTN 505 Trial. Journal of Infectious Diseases 217, 1280-1288, doi:10.1093/infdis/jiyoo8 (2018).

13 Menicucci, A. R., Sureshchandra, S., Marzi, A., Feldmann, H. \& Messaoudi, I. Transcriptomic analysis reveals a previously unknown role for CD8(+) T-cells in rVSV-EBOV mediated protection. Scientific reports 7, 919, doi:10.1038/s41598-017-01032-8 (2017).

14 Boni, C. et al. Combined GS-4774 and Tenofovir Therapy Can Improve HBV-Specific T-Cell Responses in Patients With Chronic Hepatitis. Gastroenterology 157, 227-+, doi:10.1053/j.gastro.2019.03.044 (2019).

15 Klenerman, P. \& Oxenius, A. T cell responses to cytomegalovirus. Nat Rev Immunol 16, 367-377, doi:10.1038/nri.2016.38 (2016).

16 Rouphael, N. G. et al. DNA priming and gp120 boosting induces HIV-specific antibodies in a randomized clinical trial. $J$ Clin Invest 129, 4769-4785, doi:10.1172/JCI128699 (2019).

17 Xia, M. et al. Bioengineered Norovirus S-6o Nanoparticles as a Multifunctional Vaccine Platform. ACS Nano 12, 10665-10682, doi:10.1021/acsnano.8bo2776 (2018).

18 Tan, M. \& Jiang, X. Norovirus Capsid Protein-Derived Nanoparticles and Polymers as Versatile Platforms for Antigen Presentation and Vaccine Development. Pharmaceutics 11, doi:ARTN 472 10.3390/pharmaceutics11090472 (2019).

19 Martin, J. E. et al. A SARS DNA vaccine induces neutralizing antibody and cellular immune responses in healthy adults in a Phase I clinical trial. Vaccine 26, 6338-6343, doi:10.1016/j.vaccine.2008.09.026 (2008).

20 Wong, S. S. \& Webby, R. J. Traditional and new influenza vaccines. Clin Microbiol Rev 26, 476-492, doi:10.1128/CMR.00097-12 (2013).

21 Diao, B. et al. Reduction and Functional Exhaustion of T Cells in Patients With Coronavirus Disease 2019 (COVID-19). Front Immunol 11, doi:ARTN 827 10.3389/fimmu.2020.00827 (2020).

22 Qin, C. et al. Dysregulation of immune response in patients with COVID-19 in Wuhan, China. Clin Infect Dis, doi:10.1093/cid/ciaa248 (2020).

23 Riou, J., Hauser, A., Counotte, M. J. \& Althaus, C. L. Adjusted age-specific case fatality ratio during the COVID-19 epidemic in Hubei, China, January and February 2020. medRxiv, 2020.2003.2004.20031104, doi:10.1101/2020.03.04.20031104 (2020). 
24 Rydyznski Moderbacher, C. et al. Antigen-Specific Adaptive Immunity to SARS-CoV-2 in Acute COVID-19 and Associations with Age and Disease Severity. Cell, doi:10.1016/j.cell.2020.09.038 (2020).

25 Peng, Y. et al. Broad and strong memory CD4(+) and CD8(+) T cells induced by SARS-CoV-2 in UK convalescent individuals following COVID-19. Nat Immunol 21, 1336-1345, doi:10.1038/s41590-0200782-6 (2020).

$26 \mathrm{Ni}$, L. et al. Detection of SARS-CoV-2-Specific Humoral and Cellular Immunity in COVID-19 Convalescent Individuals. Immunity 52, 971-977 e973, doi:10.1016/j.immuni.2020.04.023 (2020).

27 Habel, J. R. et al. Suboptimal SARS-CoV-2-specific CD8(+) T cell response associated with the prominent HLA-A*02:01 phenotype. Proc Natl Acad Sci U S A 117, 24384-24391, doi:10.1073/pnas.2015486117 (2020).

28 Kared, H. et al. CD8+ T cell responses in convalescent COVID-19 individuals target epitopes from the entire SARS-CoV-2 proteome and show kinetics of early differentiation. bioRxiv, doi:10.1101/2020.10.08.330688 (2020).

29 Schultheiss, C. et al. Next-Generation Sequencing of T and B Cell Receptor Repertoires from COVID19 Patients Showed Signatures Associated with Severity of Disease. Immunity, doi:10.1016/j.immuni.2020.06.024 (2020).

30 Marinaik, C. B. et al. Programming Multifaceted Pulmonary T Cell Immunity by Combination Adjuvants. Cell Rep Med 1, 100095, doi:10.1016/j.xcrm.2020.100095 (2020).

31 Sharma, M. et al. Mucosal-associated invariant T cells develop an innate-like transcriptomic program in anti-mycobacterial responses. Front Immunol, doi:10.3389/fimmu.2020.01136 (2020).

32 Huang, S. Targeting Innate-Like T Cells in Tuberculosis. Front Immunol 7, 594, doi:10.3389/fimmu.2016.00594 (2016).

33 Borg, N. A. et al. CD1d-lipid-antigen recognition by the semi-invariant NKT T-cell receptor. Nature 448, 44-49, doi:10.1038/natureo5907 (2007).

34 Armstrong, K. M., Piepenbrink, K. H. \& Baker, B. M. Conformational changes and flexibility in T-cell receptor recognition of peptide-MHC complexes. Biochem $J$ 415, 183-196, doi:10.1042/BJ20080850 (2008).

35 Millet, J. K. \& Whittaker, G. R. Physiological and molecular triggers for SARS-CoV membrane fusion and entry into host cells. Virology 517, 3-8, doi:10.1016/j.virol.2017.12.015 (2018).

36 Shang, J. et al. Cell entry mechanisms of SARS-CoV-2. Proc Natl Acad Sci U S A 117, 11727-11734, doi:10.1073/pnas.2003138117 (2020).

37 Sigal, L. J. Activation of CD8 T Lymphocytes during Viral Infections. Encyclopedia of Immunobiology, 286-290, doi:10.1016/B978-0-12-374279-7.14009-3 (2016).

38 Rosendahl Huber, S., van Beek, J., de Jonge, J., Luytjes, W. \& van Baarle, D. T cell responses to viral infections - opportunities for Peptide vaccination. Front Immunol 5, 171, doi:10.3389/fimmu.2014.00171 (2014).

39 Grifoni, A. et al. Targets of T Cell Responses to SARS-CoV-2 Coronavirus in Humans with COVID-19 Disease and Unexposed Individuals. Cell, doi:10.1016/j.cell.2020.05.015 (2020).

40 Le Bert, N. et al. SARS-CoV-2-specific T cell immunity in cases of COVID-19 and SARS, and uninfected controls. Nature, doi:10.1038/s41586-020-2550-z (2020).

41 Mateus, J. et al. Selective and cross-reactive SARS-CoV-2 T cell epitopes in unexposed humans. Science 370, 89-94, doi:10.1126/science.abd3871 (2020).

42 Robinson, J. et al. The IPD and IMGT/HLA database: allele variant databases. Nucleic Acids Res 43, D423-431, doi:10.1093/nar/gku1161 (2015).

43 Rossjohn, J. et al. T cell antigen receptor recognition of antigen-presenting molecules. Annu Rev Immunol 33, 169-200, doi:10.1146/annurev-immunol-032414-112334 (2015).

44 Lin, M. et al. Association of HLA class I with severe acute respiratory syndrome coronavirus infection. BMC Med Genet 4, 9, doi:10.1186/1471-2350-4-9 (2003).

45 Sylvester-Hvid, C. et al. SARS CTL vaccine candidates; HLA supertype-, genome-wide scanning and biochemical validation. Tissue Antigens 63, 395-400, doi:10.1111/j.0001-2815.2004.00221.x (2004).

46 Nielsen, M. et al. NetMHCpan, a Method for Quantitative Predictions of Peptide Binding to Any HLAA and -B Locus Protein of Known Sequence. PLoS One 2, doi:ARTN e796 10.1371/journal.pone.0000796 (2007).

47 Papuchova, H., Meissner, T. B., Li, Q., Strominger, J. L. \& Tilburgs, T. The Dual Role of HLA-C in Tolerance and Immunity at the Maternal-Fetal Interface. Front Immunol 10, doi:ARTN 2730 10.3389/fimmu.2019.02730 (2019). 
48 Tenzer, S. et al. Modeling the MHC class I pathway by combining predictions of proteasomal cleavage, TAP transport and MHC class I binding. Cell Mol Life Sci 62, 1025-1037, doi:10.1007/sooo18-005-4528-2 (2005).

49 Peters, B., Bulik, S., Tampe, R., Van Endert, P. M. \& Holzhutter, H. G. Identifying MHC class I epitopes by predicting the TAP transport efficiency of epitope precursors. $J$ Immunol 171, 1741-1749, doi:10.4049/jimmunol.171.4.1741 (2003).

50 Corpet, F. Multiple sequence alignment with hierarchical clustering. Nucleic Acids Res 16, 1088110890, doi:10.1093/nar/16.22.10881 (1988).

51 Sette, A. \& Sidney, J. Nine major HLA class I supertypes account for the vast preponderance of HLAA and -B polymorphism. Immunogenetics 50, 201-212, doi:10.1007/s002510050594 (1999).

52 Sidney, J., Peters, B., Frahm, N., Brander, C. \& Sette, A. HLA class I supertypes: a revised and updated classification. BMC Immunol 9, 1, doi:10.1186/1471-2172-9-1 (2008).

53 Andreatta, M., Lund, O. \& Nielsen, M. Simultaneous alignment and clustering of peptide data using a Gibbs sampling approach. Bioinformatics 29, 8-14, doi:10.1093/bioinformatics/bts621 (2013).

54 Andreatta, M., Alvarez, B. \& Nielsen, M. GibbsCluster: unsupervised clustering and alignment of peptide sequences. Nucleic Acids Res 45, W458-W463, doi:10.1093/nar/gkx248 (2017).

55 Falk, K. et al. Peptide motifs of HLA-A1, -A11, -A31, and -A33 molecules. Immunogenetics 4o, 238241, doi:10.1007/BFo0167086 (1994).

56 Falk, K., Rotzschke, O., Stevanovic, S., Jung, G. \& Rammensee, H. G. Allele-specific motifs revealed by sequencing of self-peptides eluted from MHC molecules. Nature 351, 290-296, doi:10.1038/351290ao (1991).

57 Maier, R. et al. Peptide Motifs of Hla-A3, Hla-A24, and Hla-B7 Molecules as Determined by Pool Sequencing. Immunogenetics 40, 306-308, doi:Doi 10.1007/Bfoo189978 (1994).

58 Barber, L. D. et al. Overlap in the repertoires of peptides bound in vivo by a group of related class I HLA-B allotypes. Curr Biol 5, 179-190, doi:10.1016/so960-9822(95)0o039-x (1995).

59 Arnett, K. L., Huang, W., Valiante, N. M., Barber, L. D. \& Parham, P. The Bw4/Bw6 difference between HLA-B*0802 and HLA-B*0801 changes the peptides endogenously bound and the stimulation of alloreactive T cells. Immunogenetics 48, 56-61, doi:10.1007/so02510050400 (1998).

60 Falk, K. et al. Peptide motifs of HLA-B35 and -B37 molecules. Immunogenetics 38, 161-162, doi:10.1007/BFo0190906 (1993).

61 Sanchez-Mazas, A. HLA studies in the context of coronavirus outbreaks. Swiss Med Wkly 150, w20248, doi:10.4414/smw.2020.20248 (2020).

62 Doytchinova, I. A., Guan, P. P. \& Flower, D. R. Identifiying human MHC supertypes using bioinformatic methods. J Immunol 172, 4314-4323, doi:DOI 10.4049/jimmunol.172.7.4314 (2004).

63 Rapin, N., Lund, O., Bernaschi, M. \& Castiglione, F. Computational Immunology Meets Bioinformatics: The Use of Prediction Tools for Molecular Binding in the Simulation of the Immune System. PLoS One 5, doi:ARTN e9862 10.1371/journal.pone.0009862 (2010).

64 Maiers, M., Gragert, L. \& Klitz, W. High-resolution HLA alleles and haplotypes in the United States population. Hum Immunol 68, 779-788, doi:10.1016/j.humimm.2007.04.005 (2007).

65 Doolan, D. L. et al. Degenerate cytotoxic T cell epitopes from P. falciparum restricted by multiple HLA-A and HLA-B supertype alleles. Immunity 7, 97-112, doi:10.1016/s1074-7613(00)80513-0 (1997).

66 Campbell, K. M., Steiner, G., Wells, D. K., Ribas, A. \& Kalbasi, A. Prediction of SARS-CoV-2 epitopes across 9360 HLA class I alleles. bioRxiv, doi:10.1101/2020.03·30.016931 (2020).

67 Pierini, F. \& Lenz, T. L. Divergent Allele Advantage at Human MHC Genes: Signatures of Past and Ongoing Selection. Mol Biol Evol 35, 2145-2158, doi:10.1093/molbev/msy116 (2018).

68 Arora, J. et al. HLA Heterozygote Advantage against HIV-1 Is Driven by Quantitative and Qualitative Differences in HLA Allele-Specific Peptide Presentation. Mol Biol Evol 37, 639-650, doi:10.1093/molbev/msz249 (2020).

69 Jeffery, K. J. et al. The influence of HLA class I alleles and heterozygosity on the outcome of human T cell lymphotropic virus type I infection. J Immunol 165, 7278-7284, doi:10.4049/jimmunol.165.12.7278 (2000).

70 Hoof, I. et al. NetMHCpan, a method for MHC class I binding prediction beyond humans. Immunogenetics 61, 1-13, doi:10.1007/s00251-008-0341-z (2009).

71 Jurtz, V. et al. NetMHCpan-4.0: Improved Peptide-MHC Class I Interaction Predictions Integrating Eluted Ligand and Peptide Binding Affinity Data. J Immunol 199, 3360-3368, doi:10.4049/jimmunol.1700893 (2017). 
72 Kesmir, C., Nussbaum, A. K., Schild, H., Detours, V. \& Brunak, S. Prediction of proteasome cleavage motifs by neural networks. Protein Eng 15, 287-296, doi:10.1093/protein/15.4.287 (2002).

73 Nielsen, M., Lundegaard, C., Lund, O. \& Kesmir, C. The role of the proteasome in generating cytotoxic T-cell epitopes: insights obtained from improved predictions of proteasomal cleavage.

Immunogenetics 57, 33-41, doi:10.1007/s00251-005-0781-7 (2005).

74 Mancini, E. et al. A study on the dynamics of temporary HIV treatment to assess the controversial outcomes of clinical trials: An in-silico approach. PLoS One 13, e0200892, doi:10.1371/journal.pone.0200892 (2018). 
Fig. 1

A. Numbers of predicted SARS-CoV-2 peptide ligands for major HLA class I proteins

\begin{tabular}{|c|c|c|c|c|c|c|}
\hline $\begin{array}{c}\text { SARS-CoV-2 } \\
\text { proteins } \\
\text { ( residues) }\end{array}$ & $\begin{array}{c}\mathrm{S} \\
(1273)\end{array}$ & $\begin{array}{c}\mathrm{M} \\
(222)\end{array}$ & $\begin{array}{c}\mathrm{N} \\
(419)\end{array}$ & $\begin{array}{c}\text { ORF1ab } \\
(6954)\end{array}$ & $\begin{array}{c}\text { ORF3a } \\
(275)\end{array}$ & $\begin{array}{c}\text { ORF8 } \\
(119)\end{array}$ \\
\hline HLA-A*01:01 & 26 & 6 & 4 & 137 & 10 & 2 \\
\hline HLA-A*02:01 & 91 & 41 & 8 & 656 & 48 & 8 \\
\hline HLA-A*03:01 & 57 & 9 & 13 & 409 & 14 & 1 \\
\hline HLA-A*11:01 & 107 & 23 & 19 & 616 & 22 & 1 \\
\hline HLA-A*24:02 $^{*}$ & 53 & 20 & 3 & 286 & 16 & 4 \\
\hline HLA-B*07:02 & 17 & 6 & 18 & 158 & 6 & 4 \\
\hline HLA-B*08:01 & 34 & 9 & 8 & 171 & 9 & 5 \\
\hline HLA-B* $35: 01$ & 67 & 14 & 13 & 334 & 14 & 3 \\
\hline HLA-B $^{*} 40: 01$ & 19 & 5 & 1 & 170 & 5 & 2 \\
\hline HLA-B $^{*} 44: 03$ & 16 & 4 & 3 & 119 & 9 & 0 \\
\hline
\end{tabular}

B. \% numbers of predicted peptide ligands for HLA class I proteins over the numbers of amino acid sequence for SARS-CoV-2 proteins

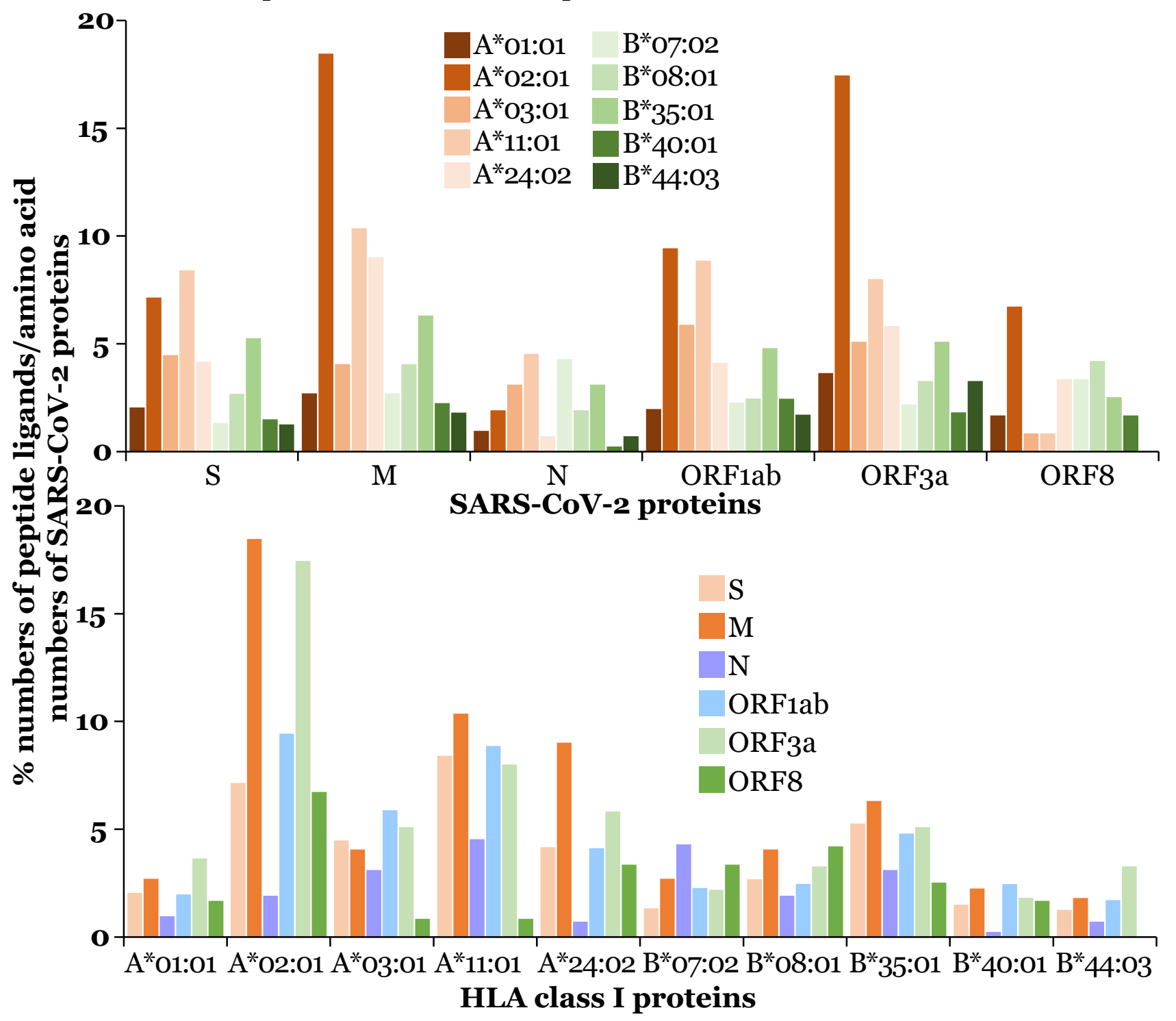




\section{Fig. 2}

A. Overlaps of predicted SARS-CoV-2 peptide ligands for HLA-A proteins
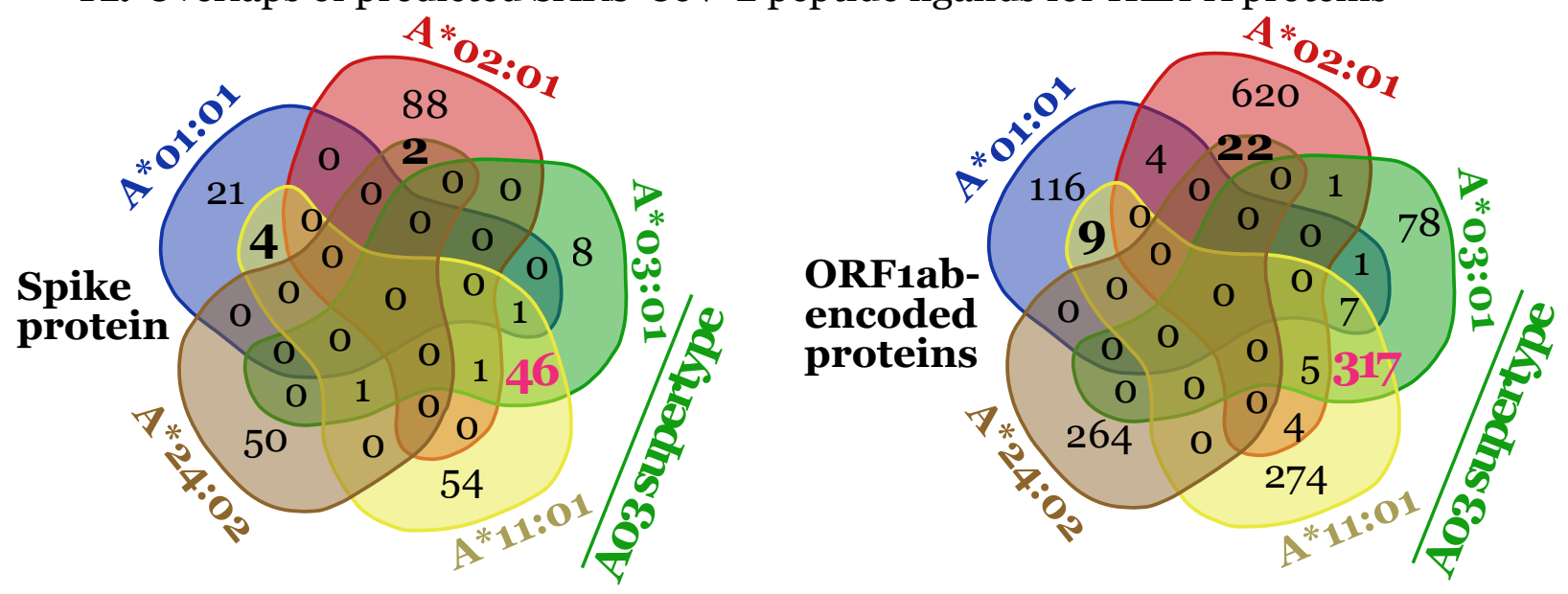

B. Overlaps of predicted SARS-CoV-2 peptide ligands for HLA-B proteins
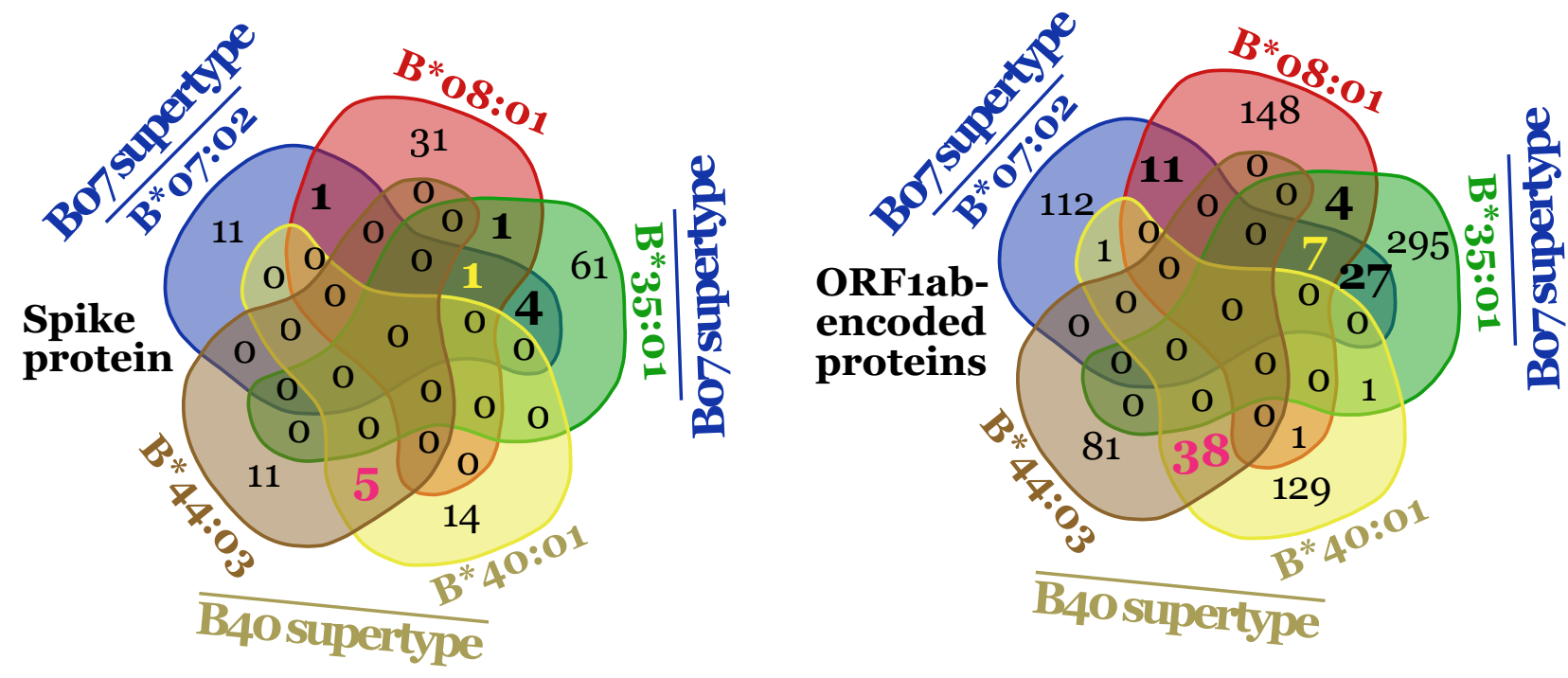


\section{Fig. 3}

A. Enriched residues in predicted SARS-CoV-2 peptide ligands for HLA-A proteins
A*01:01
$\mathrm{A}^{*}$ 02:01
$\frac{\text { A03supertype }}{\mathrm{A}^{* 03: 01} \quad \mathrm{~A}^{* 11: 01}}$

A*24:02

\# residues $12 \underline{3} 456 \underline{789} 12345 \underline{6} 78 \mathbf{9}$

123456789

$12 \underline{3} 456 \underline{7} 89$

123456789

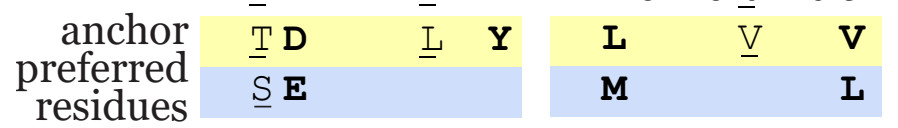

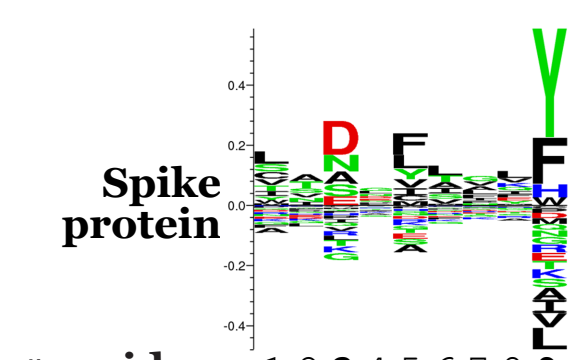

\# residues $12345678 \mathbf{9}^{-022} 12345678$ 命
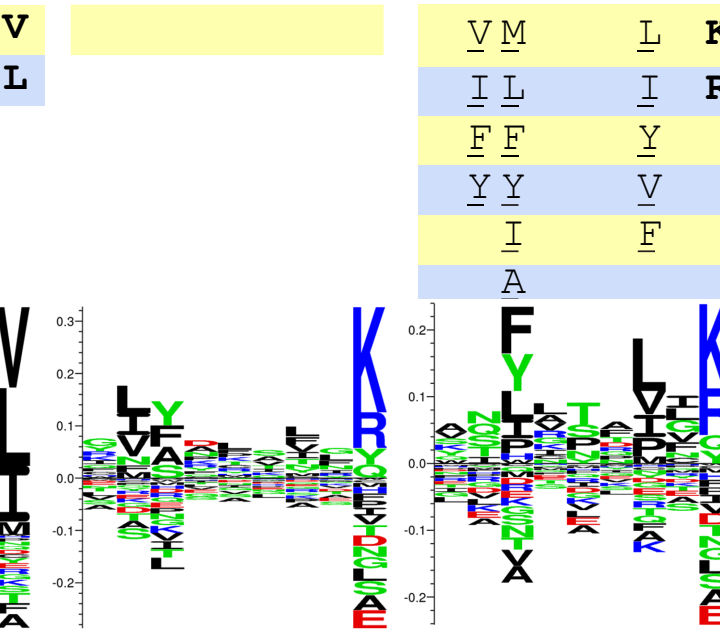

$\begin{array}{ll}\mathbf{K} & \mathbf{Y} \\ \mathbf{R} & \mathbf{F}\end{array}$

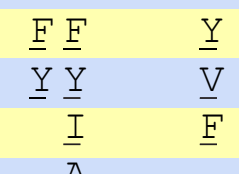

I
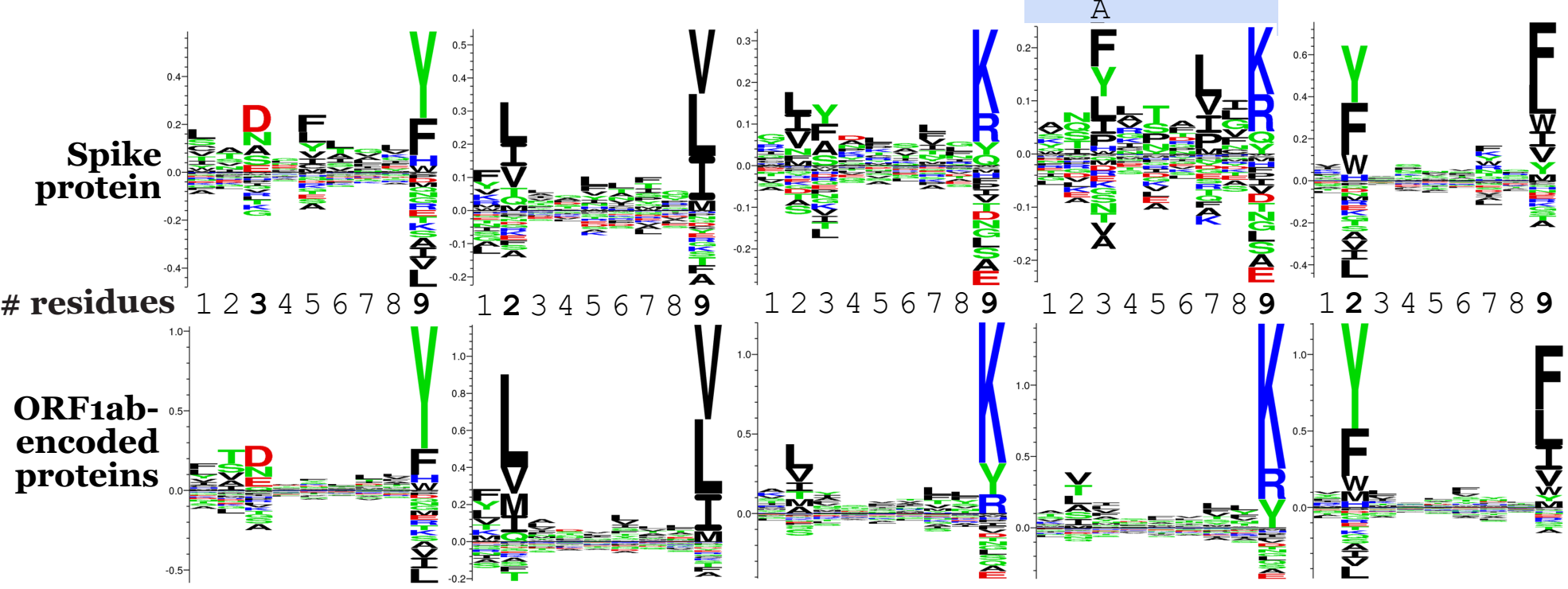

123456789

B. Enriched residues in predicted SARS-CoV-2 peptide ligands for HLA-B proteins

$\frac{\text { Bo7supertype }}{\text { B }^{*} \mathbf{0 7 : 0 2}}$

\# residues 123456789

anchor $\mathbf{P}$

preferred

residues
L

$B^{* 08: 01}$

\begin{abstract}
123456789
\end{abstract}
$\frac{\text { Bo7supertype }}{\text { B* } 35: 01^{*}}$

123456789

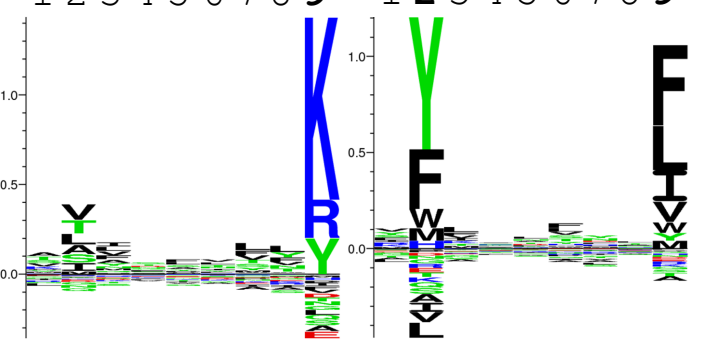


A. Numbers of predicted SARS-CoV-2 peptide ligands for HLA class I proteins based on proteasome digestion, TAP transportation, and peptide binding affinity.

\begin{tabular}{|l|c|c|c|c|c|c|}
\hline $\begin{array}{c}\text { SARS-CoV-2 } \\
\text { proteins }\end{array}$ & $\begin{array}{c}\mathrm{S} \\
(1273)\end{array}$ & $\begin{array}{c}\mathrm{M} \\
(222)\end{array}$ & $\begin{array}{c}\mathrm{N} \\
(419)\end{array}$ & $\begin{array}{c}\text { ORF1ab } \\
(6954)\end{array}$ & $\begin{array}{c}\text { ORF3a } \\
(275)\end{array}$ & $\begin{array}{c}\text { ORF8 } \\
(119)\end{array}$ \\
\hline HLA-A*02:01 & 91 & 41 & 8 & 656 & 48 & 8 \\
\hline HLA-A*02:02 & 173 & 55 & 12 & 1104 & 72 & 10 \\
\hline HLA-A*11:01 & 107 & 23 & 19 & 616 & 22 & 1 \\
\hline HLA-A*25:01 & 4 & 0 & 1 & 28 & 3 & 0 \\
\hline HLA-B*07:02 & 17 & 6 & 18 & 158 & 6 & 4 \\
\hline HLA-B*07:03 & 18 & 7 & 11 & 171 & 5 & 1 \\
\hline HLA-B*15:01 & 125 & 31 & 19 & 741 & 26 & 11 \\
\hline HLA-B*15:02 & 65 & 19 & 11 & 393 & 13 & 7 \\
\hline HLA-B*15:03 & 274 & 47 & 44 & 1538 & 64 & 29 \\
\hline HLA-B* $46: 01$ & 0 & 1 & 0 & 2 & 0 & 0 \\
\hline
\end{tabular}

B. \% numbers of predicted peptide ligands for HLA-A proteins over the numbers of amino acid residues from SARS-CoV-2 proteins

HLA-A

Spike
protein

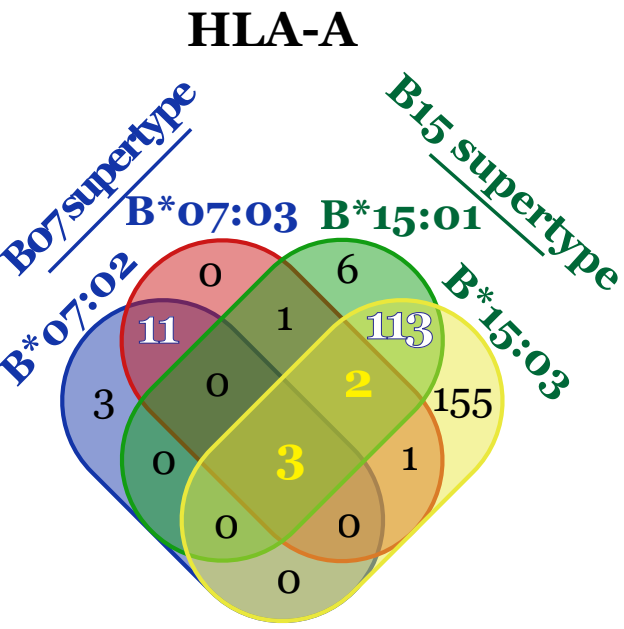

HLA-A \& HLA-B

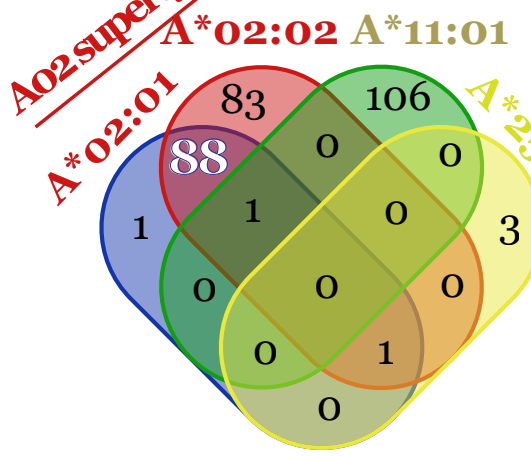

ORF1ab-

encoded

proteins
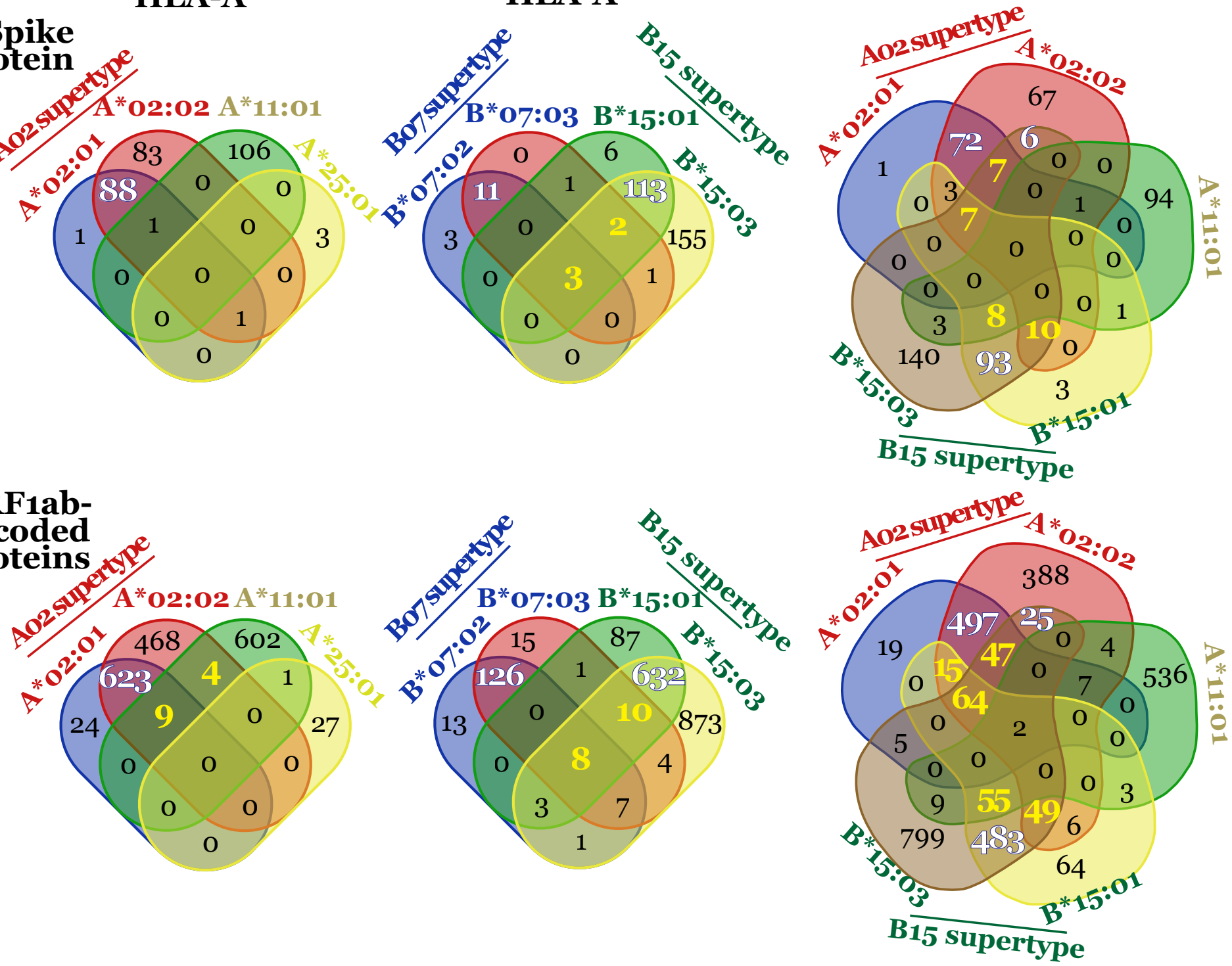


\section{Fig. 5}

A. Venn diagrams of overlapped SARS-CoV-2 peptide ligands for major HLA class I A and B proteins

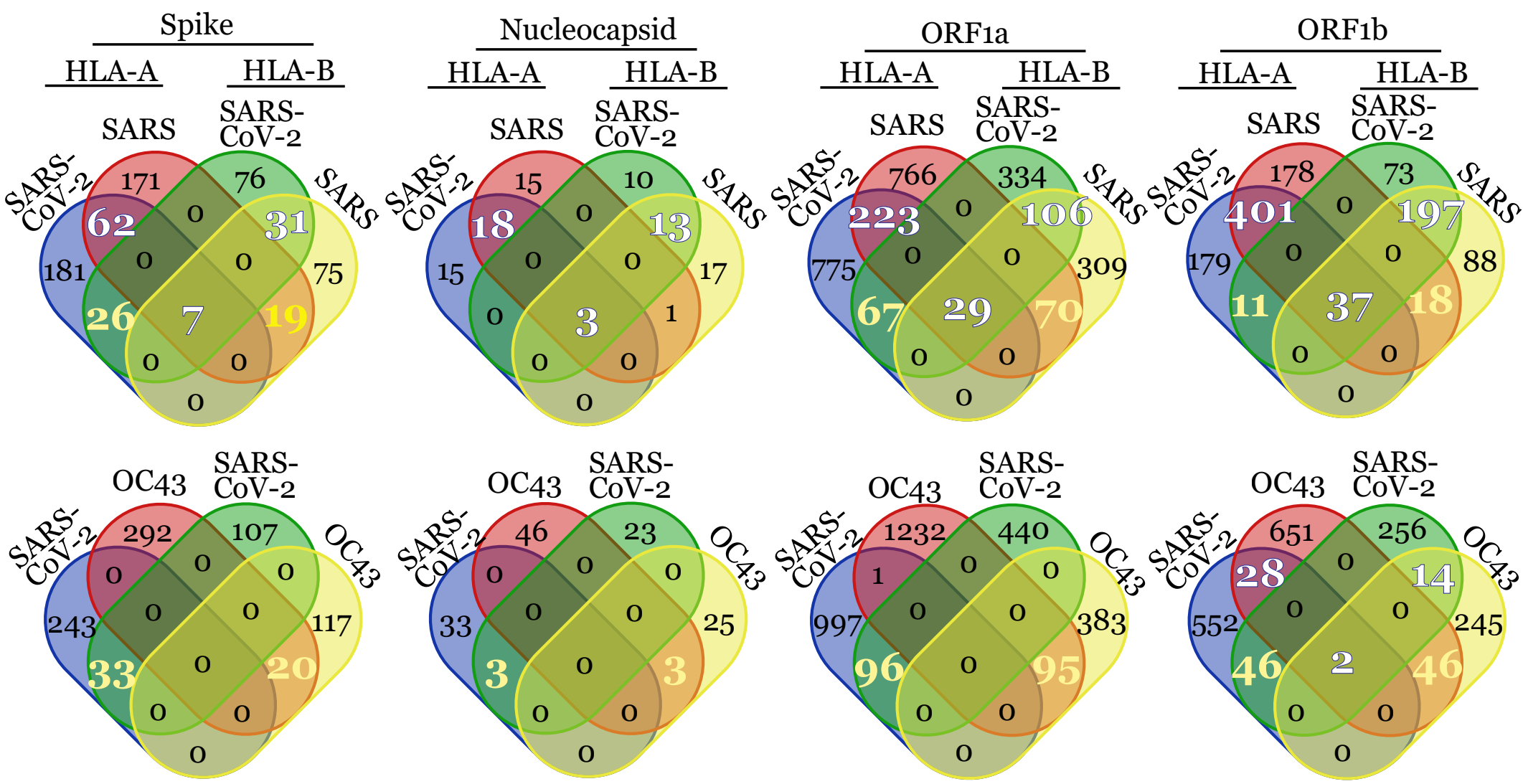

B. \% numbers of overlapped peptide ligands predicted from SARS-CoV-2 and other coronavirus proteins for major HLA class I A and B proteins

\begin{tabular}{|c|c|c|c|c|c|}
\hline \multirow{2}{*}{$\begin{array}{l}\text { Ligand binding } \\
\text { with }\end{array}$} & \multirow{2}{*}{$\begin{array}{l}\text { Coronaviruses to } \\
\text { compare with } \\
\text { SARS-CoV-2 }\end{array}$} & \multicolumn{4}{|c|}{ \% overlapped SARS-CoV-2 peptides } \\
\hline & & S & $\mathrm{N}$ & ORF1a & ORF1b \\
\hline \multirow{2}{*}{$\begin{array}{c}\text { HLA-A* } 01: 01, \\
A^{*} 02: 01, A^{*}: 03: 01, \\
A^{*} 11: 01, \text { and } \\
A^{*} 24: 02\end{array}$} & SARS & 25.00 & 58.33 & 23.03 & 69.75 \\
\hline & $\mathrm{HCoV}-\mathrm{OC} 43$ & o & 0 & 0.09 & $4 \cdot 78$ \\
\hline \multirow{2}{*}{$\begin{array}{c}\text { HLA-B*07:02, } \\
\text { B* }^{*} \text { 8:01, B*35:01, } \\
\mathrm{B}^{*} 40: 01 \text {, and } \\
\mathrm{B}^{*} 44: 03\end{array}$} & SARS & 27.14 & 61.24 & 25.19 & 73.58 \\
\hline & $\mathrm{HCoV}-\mathrm{OC} 43$ & o & o & o & 5.03 \\
\hline
\end{tabular}




\section{Fig. 6}

A. Immune simulation using different numbers of predicted peptide ligands for HLA-A*02:01
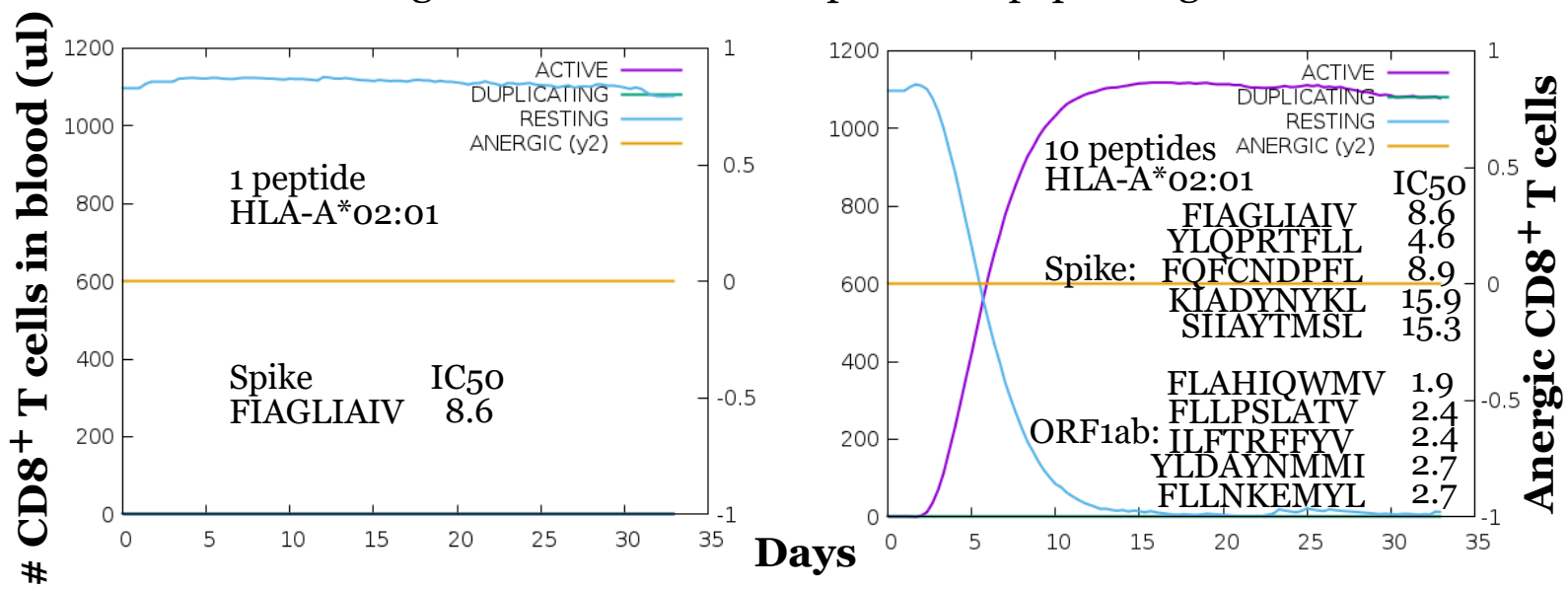

B. Immune simulation using peptides with different binding affinity to HLA-A*02:01
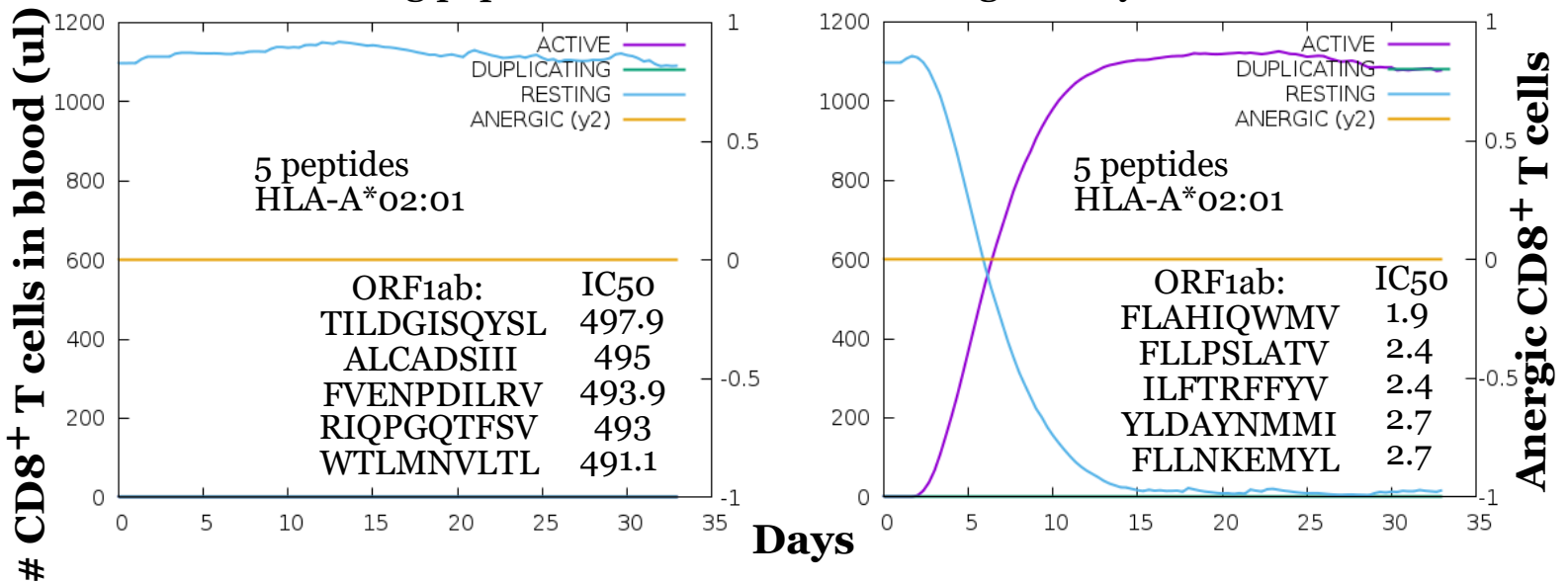

C. Immune simulation of antigen presentation mediated by heterozygous and paralogous major HLA class I A and B proteins with predicted peptide ligands from the spike protein as an exmaple
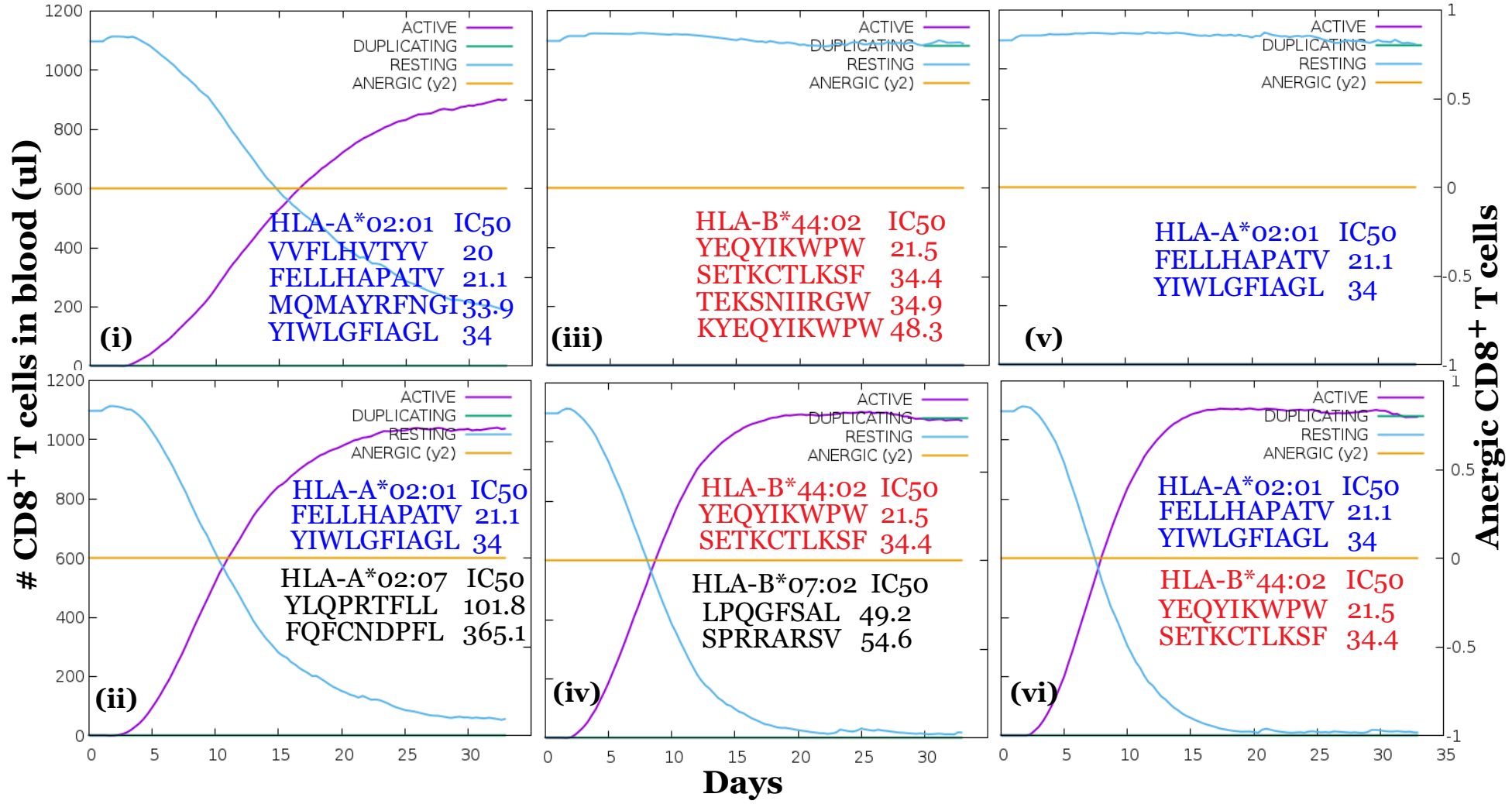
A. Immune simulation of antigen presentation mediated by a weak peptide binder and a heterozygous or paralogous HLA class I protein.
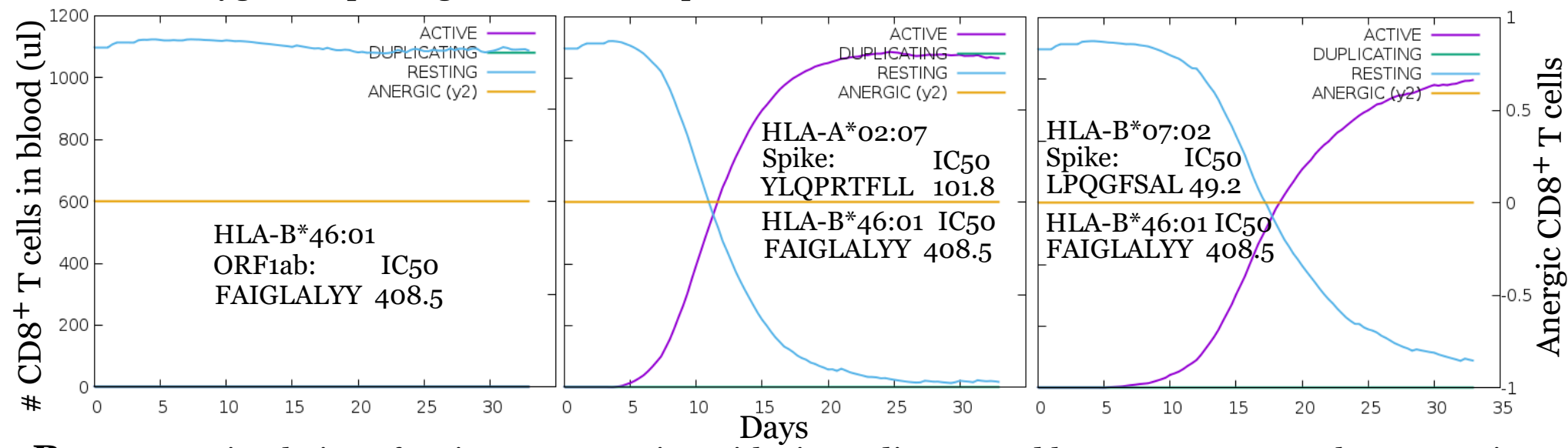

B. Immune simulation of antigen presentation with virus adjuvant and heterozygous HLA class I proteins
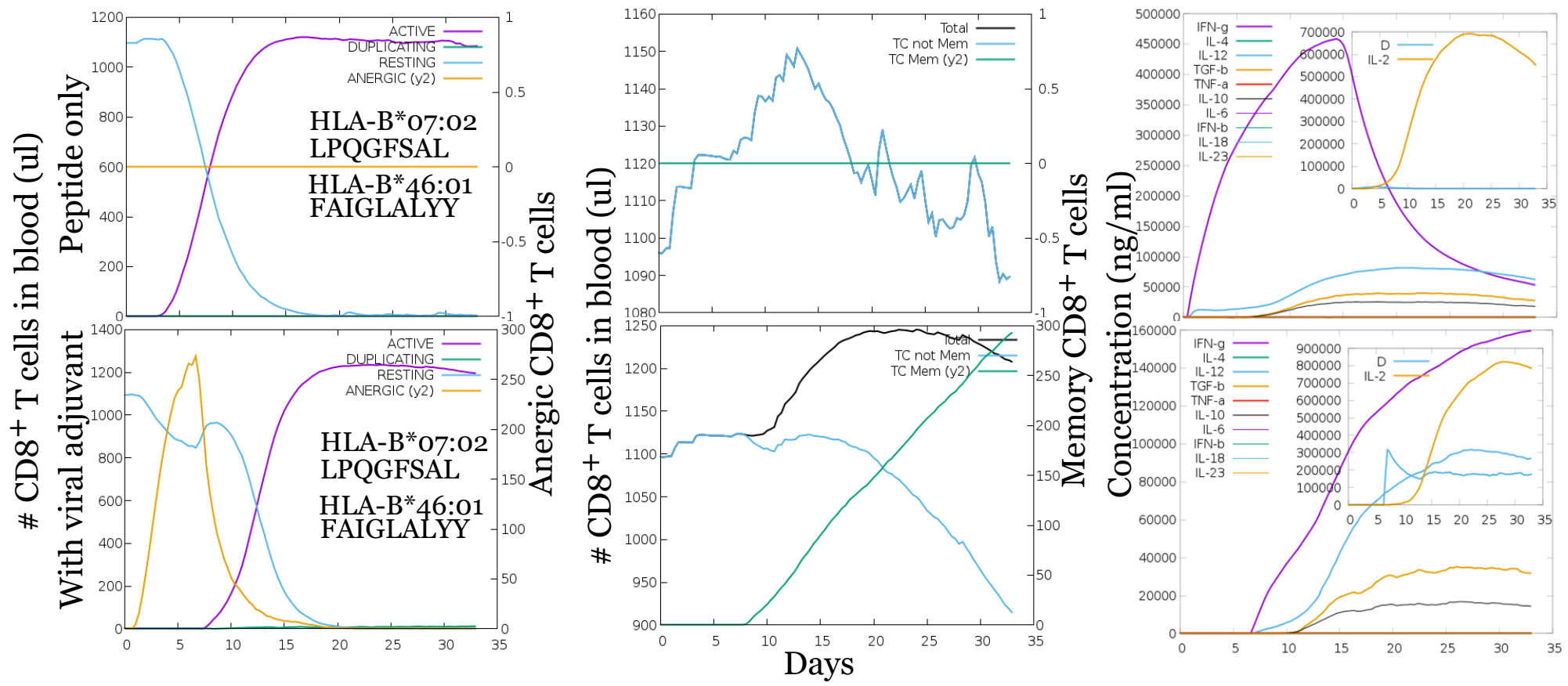

C. Immune simulation of antigen presentation with virus adjuvant and paralogous HLA class I proteins
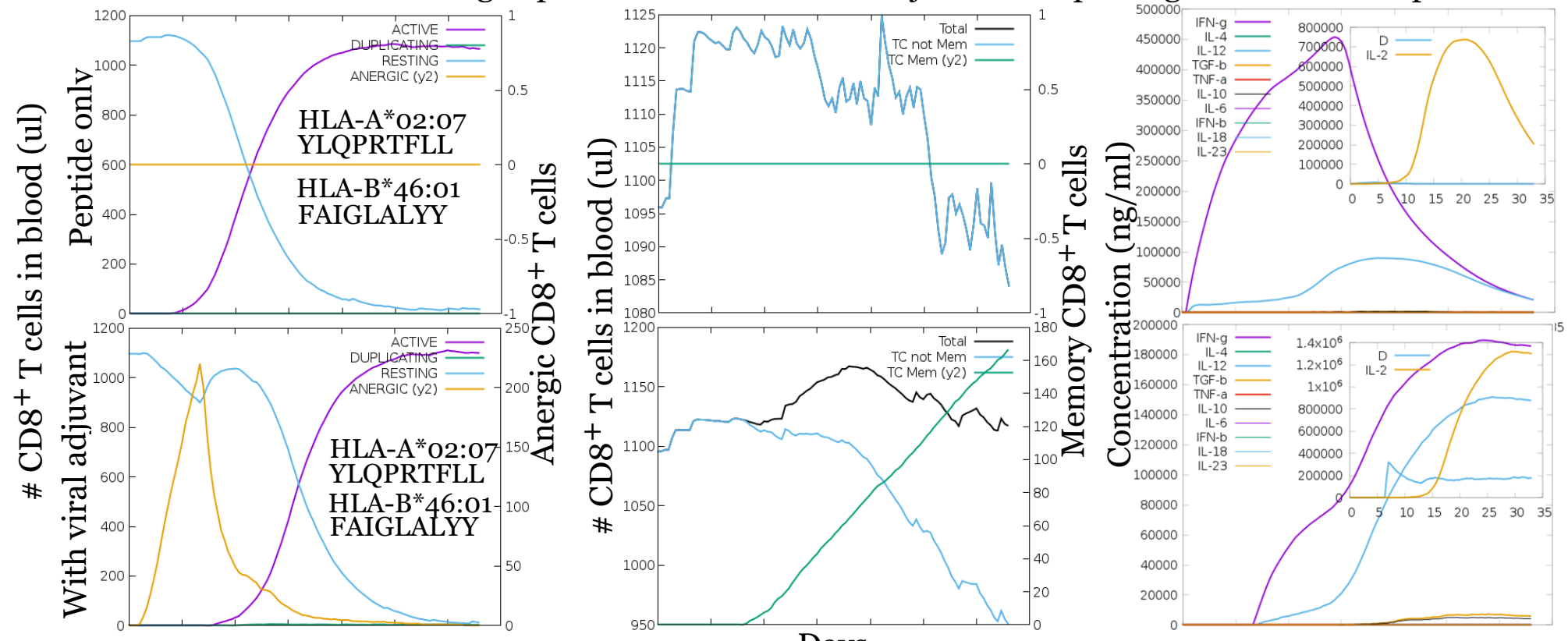

Days 


\section{Supplementary Information}

2

3

4

HLA class I genotypes customize vaccination strategies in immune simulation to combat COVID-19

7

8 Shouxiong Huang ${ }^{1,2 *} \&$ Ming Tan 3

9

10

${ }^{1}$ Department of Environmental and Public Health Sciences, University of Cincinnati College of

11 Medicine, Cincinnati, OH 45249, USA

$12{ }^{2}$ Immunology Graduate Program, Cincinnati Children's Hospital Medical Center, Cincinnati, $\mathrm{OH}$

13 45229, USA

14 3Division of Infectious Diseases, Cincinnati Children's Hospital Medical Center, Cincinnati, OH

15 45229, USA

16

17

18

Keywords: Binding affinity, human leukocyte antigen class I, CD8+ T cells, coronavirus disease 2019 (COVID-19), custom vaccination

20

21

*Corresponding author. E-mail address: Shouxiong.huang@uc.edu

22

23

24

25

26

27

28

29 
A. Numbers of predicted SARS-CoV-2 peptides for major HLA class I proteins based on peptide binding affinity.

\begin{tabular}{|c|c|c|c|c|c|c|}
\hline $\begin{array}{c}\text { SARS-CoV-2 } \\
\text { proteins }\end{array}$ & $\begin{array}{c}\mathrm{S} \\
(1273)\end{array}$ & $\begin{array}{c}\mathrm{M} \\
(222)\end{array}$ & $\begin{array}{c}\mathrm{N} \\
(419)\end{array}$ & $\begin{array}{c}\text { ORF1ab } \\
(6954)\end{array}$ & $\begin{array}{c}\text { ORF3a } \\
(275)\end{array}$ & $\begin{array}{c}\text { ORF8 } \\
(119)\end{array}$ \\
\hline HLA-A*01:01 & 28 & 6 & 4 & 146 & 13 & 2 \\
\hline HLA-A*02:01 & 102 & 59 & 8 & 818 & 53 & 13 \\
\hline HLA-A*03:01 & 63 & 10 & 18 & 481 & 16 & 1 \\
\hline HLA-A*11:01 & 114 & 25 & 25 & 716 & 24 & 1 \\
\hline HLA-A $^{*} 24: 02$ & 54 & 22 & 3 & 288 & 16 & 4 \\
\hline HLA-B*07:02 & 28 & 7 & 20 & 189 & 7 & 4 \\
\hline HLA-B $08: 01$ & 36 & 11 & 8 & 194 & 11 & 5 \\
\hline HLA-B $35: 01$ & 74 & 15 & 13 & 363 & 16 & 3 \\
\hline HLA-B $^{*} 40: 01$ & 19 & 5 & 1 & 186 & 5 & 2 \\
\hline HLA-B $^{*} 44: 03$ & 17 & 4 & 3 & 129 & 9 & O \\
\hline
\end{tabular}

B. Alignment of selected major HLA-A and HLA-B alleles in US populations

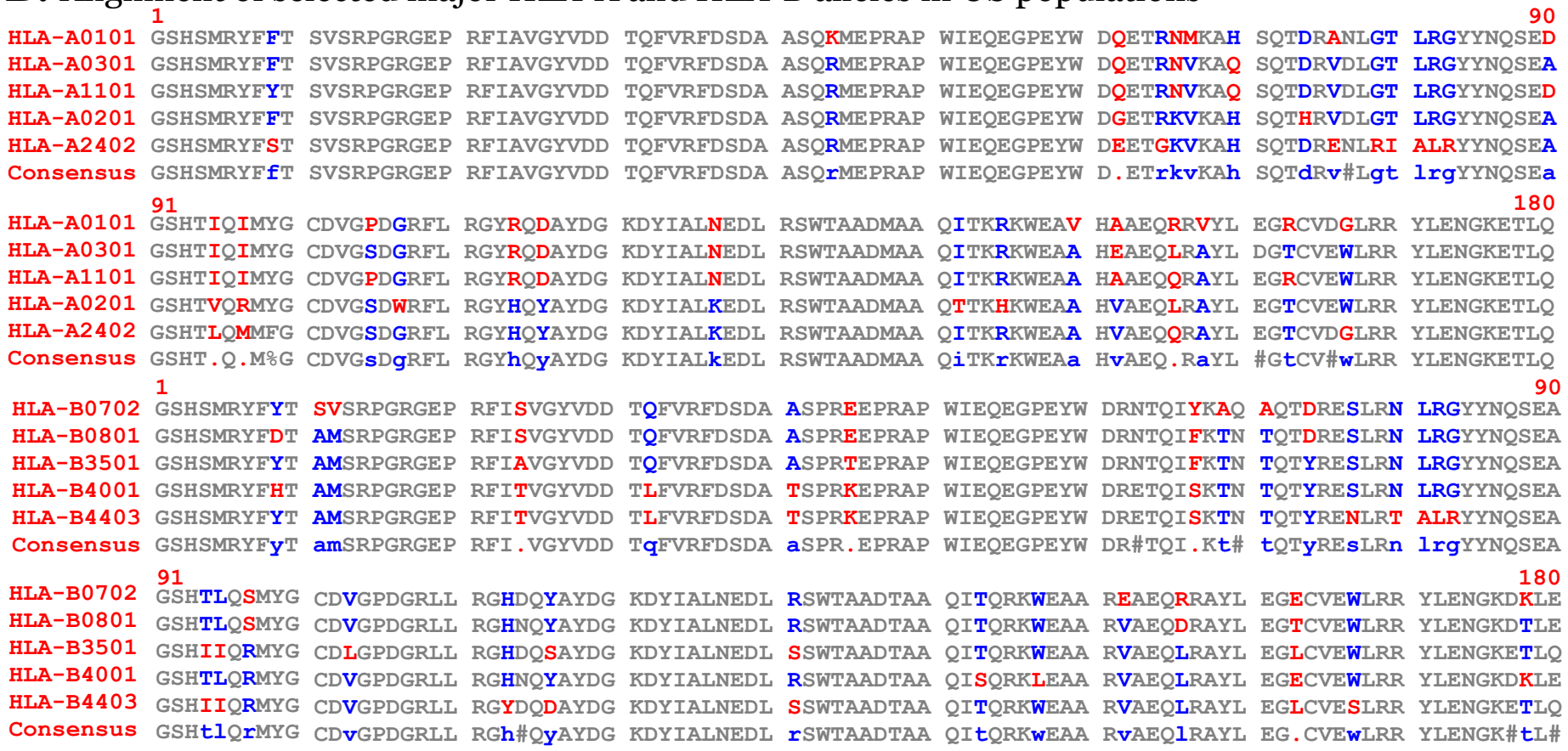

C. Sequence similarity of $\alpha 1$ and $\alpha 2$ domains of selected major HLA-A and HLA-B alleles in US populations

\begin{tabular}{|c|c|c|c|c|c|c|c|c|c|c|}
\hline & $A^{*} 01: 01$ & $A^{*} 02: 01$ & $A^{*} 03: 01$ & $A^{*}$ 11:01 & $A^{*} 24: 02$ & $\mathbf{B}^{* 07: 02}$ & $B^{*} 08: 01$ & $B^{*} 35: 01$ & $B^{*} 40: 01$ & B*44:03 \\
\hline$A^{*}$ 01:01 & 100 & 86.81 & 91.76 & 93.96 & 86.26 & 80.22 & 78.57 & 78.57 & 75.82 & 78.57 \\
\hline$A^{*} 02: 01$ & & 100 & 92.31 & 90.11 & 89.01 & 80.22 & 80.22 & 80.22 & 78.57 & 78.57 \\
\hline$A^{*} 03: 01$ & & & 100 & 96.15 & $87 \cdot 36$ & 82.97 & 81.32 & 81.32 & 78.57 & 80.22 \\
\hline$A^{*} 11: 01$ & & & & 100 & 86.81 & 83.52 & 81.32 & 81.87 & 78.57 & 80.77 \\
\hline$A^{*} 24: 02$ & & & & & 100 & 80.77 & 80.77 & 78.57 & 77.47 & 80.77 \\
\hline B*07:02 & & & & & & 100 & 93.41 & 88.46 & 89.56 & $83.5^{2}$ \\
\hline$B^{*} 08: 01$ & & & & & & & 100 & 91.76 & 92.31 & 86.26 \\
\hline$B^{*} 35: 01$ & & & & & & & & 100 & 89.56 & 91.76 \\
\hline$B * 40: 01$ & & & & & & & & & 100 & 89.56 \\
\hline$B^{*} 44: 03$ & & & & & & & & & & 100 \\
\hline
\end{tabular}

Fig. S1. Predicted SARS-CoV-2 peptide ligands only based on binding affinity to HLA class I proteins and sequence similarity in HLA class I proteins. Numbers show predicted peptide ligands from immunogenic SARS-CoV-2 proteins only based on binding affinity to major HLA class I proteins (A). Alignment of $\alpha 1$ and $\alpha 2$ domains of these HLA-A and HLA-B proteins was performed using Clustal Omega program with residues colored in grey (identical residues), red (highly diverse), and blue (less diverse) (B). \% identical residues are listed (C). 
A. Alignment of selected major HLA-A and HLA-B alleles potentially associated with SARS

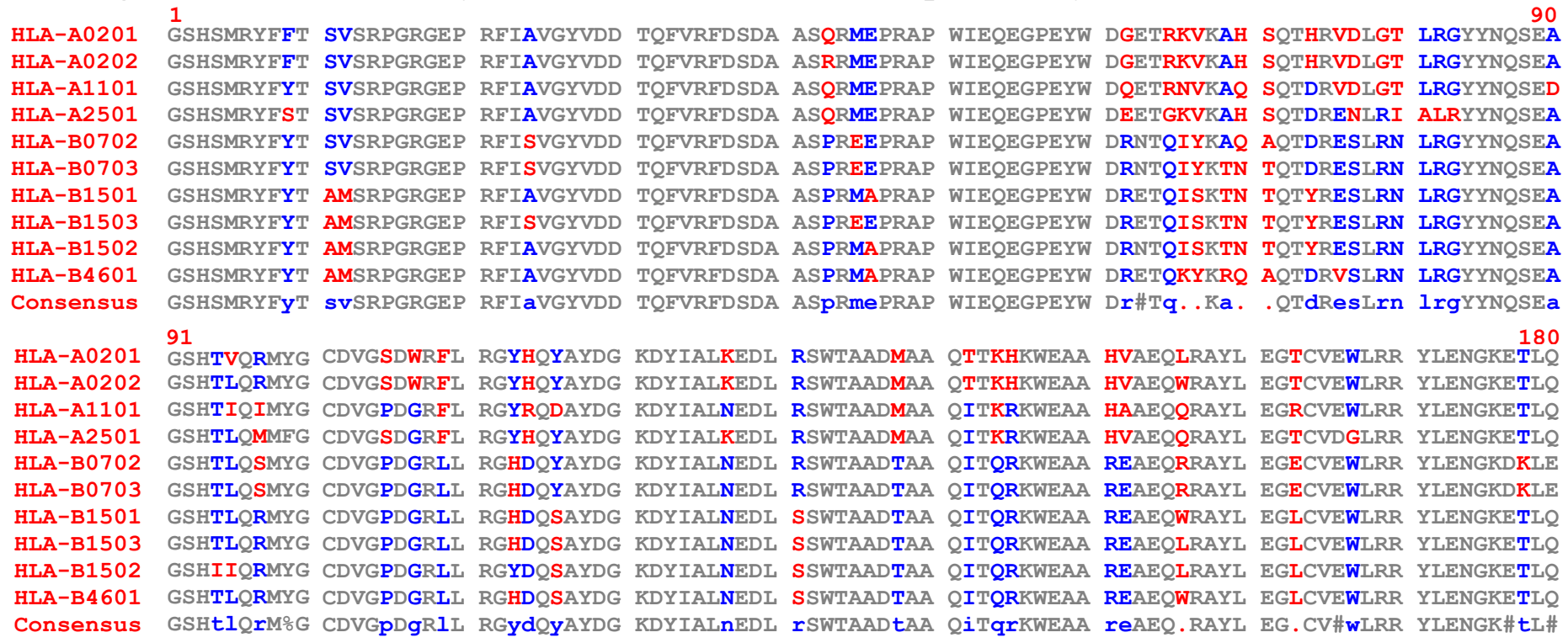

B. Sequence similarity of $\alpha 1$ and $\alpha 2$ domains of selected HLA-A and HLA-B alleles associated with SARS

\begin{tabular}{|c|c|c|c|c|c|c|c|c|c|c|}
\hline & $A^{*} 02: 01$ & $A^{*} 02: 02$ & $A^{*} 11: 01$ & $A^{*} 25: 01$ & B $^{*} 07: 02$ & $\mathbf{B}^{*} \mathbf{0 7 : 0 3}$ & $B^{*} 15: 01$ & $B^{*} 15: 02$ & $B^{*} 15: 03$ & $B^{*} 46: 01$ \\
\hline$A^{*} 02: 01$ & 100 & 98.35 & 90.11 & 89.01 & 80.22 & 79.67 & 80.77 & 80.77 & 80.77 & 81.87 \\
\hline$A^{*} 02: 02$ & & 100 & 89.56 & 89.01 & 80.77 & 80.22 & 81.87 & 80.22 & 80.77 & 82.97 \\
\hline $\mathbf{A}^{*} 11: 01$ & & & 100 & 86.81 & 83.52 & 82.42 & 82.97 & 82.97 & 82.42 & 84.62 \\
\hline$A^{*} 25: 01$ & & & & 100 & 80.77 & 80.22 & 80.22 & 79.12 & 79.67 & 80.77 \\
\hline $\mathbf{B}^{*} \mathbf{0 7 : 0 2}$ & & & & & 100 & 98.35 & 89.56 & 88.46 & 91.21 & 90.66 \\
\hline $\mathbf{B}^{*} \mathbf{0 7 : 0 3}$ & & & & & & 100 & 91.21 & 90.11 & 92.86 & 89.56 \\
\hline$B^{*} 15: 01$ & & & & & & & 100 & 97.25 & 97.8 & 96.15 \\
\hline$B^{*} 15: 02$ & & & & & & & & 100 & 96.15 & 93.41 \\
\hline$B^{*} 15: 03$ & & & & & & & & & 100 & 93.96 \\
\hline$B * 46: 01$ & & & & & & & & & & 100 \\
\hline
\end{tabular}

Fig. S2. Alignment and sequence identity of HLA class I proteins relevant or irrelevant to SARS infections. A. alignment of $\alpha 1$ and $\alpha 2$ domains for HLA class I proteins that were indicated relevant or irrelevant to the severity or protection of SARS. Residues are colored in grey (identical residues), red (highly diverse), and blue (less diverse). B. \% identical residues are listed. 
A. Sequence similarlity of spike protein sequences between SARS-CoV-2, SARS, and HCoV-OC43

\begin{tabular}{|c|c|c|c|} 
Spike & SARS-CoV-2 & SARS & HCoV-OC43 \\
\hline SARS-CoV-2 & 100 & 77.54 & 33.06 \\
SARS & & 100 & 33.17 \\
HCoV-OC43 & & & 100 \\
\hline
\end{tabular}

B. Alignment of spike protein sequences between SARS-CoV-2 and SARS

C. Alignment of spike protein sequences between SARS-CoV-2 and HCoV-OC43

Fig. S3. Alignment and sequence identity of spike protein between SARS-CoV-2 and other coronavirus. A. \% sequence identity is listed. B. alignment of S protein between SARS-CoV-2 and SARS. C. alignment of S protein between SARS-CoV-2 and HCoV-OC43. 


\section{Fig. S4}

A. Sequence similarity of ORF1b-encoded protein sequences between SARS-CoV-2, SARS, and HCoV-OC43

\begin{tabular}{|c|c|c|c|}
\hline ORF1b-encoded proteins & SARS-CoV-2 & SARS & HCoV-OC43 \\
\hline SARS-CoV-2 & 100 & $95 \cdot 37$ & 62.32 \\
\hline SARS & & 100 & 62.82 \\
\hline HCoV-OC43 & & & 100 \\
\hline
\end{tabular}

B. Alignment of ORF1b-encoded protein sequences between SARS-CoV-2 and SARS

Fig. S4. Alignment and sequence identity of ORF1b-endoced proteins between SARS-CoV-2 and other coronaviruses. A. \% sequence identity is listed. B. alignment of ORF1b-endoced proteins between SARS-CoV-2 and SARS. C. alignment of ORF1b-endoced proteins between SARS-CoV-2 and $\mathrm{HCoV}-\mathrm{OC} 43$. 


\section{Fig. S4}

\section{Alignment of ORF1b-encoded protein sequences between SARS-CoV-2 and SARS}

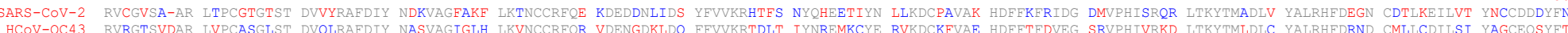

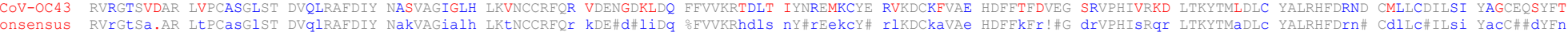

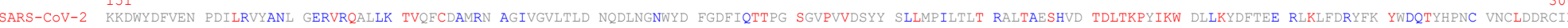

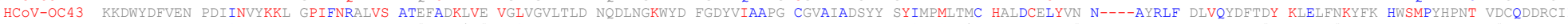

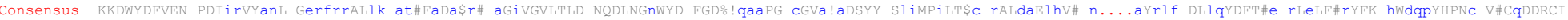

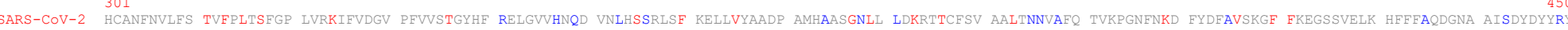

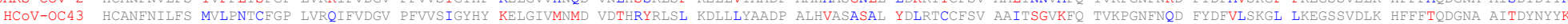

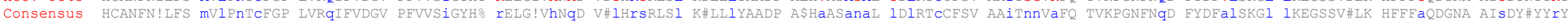
451

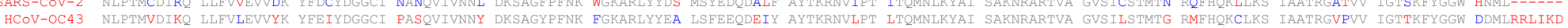

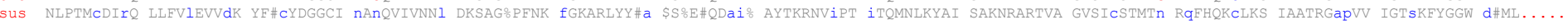
601

SARS-COV-2

751

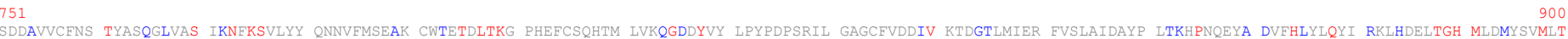

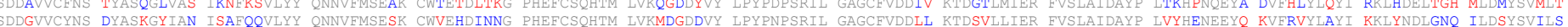

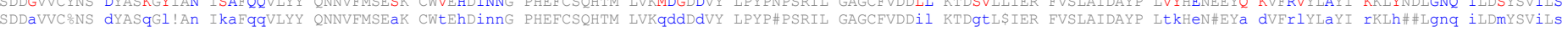
901

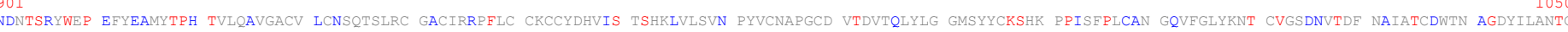

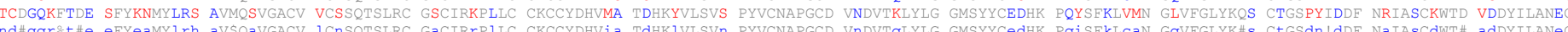
1051

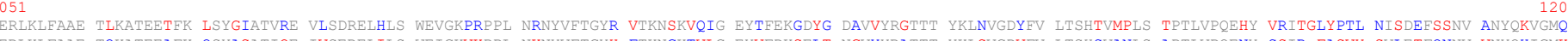

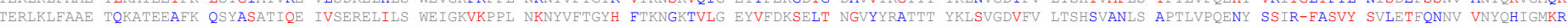

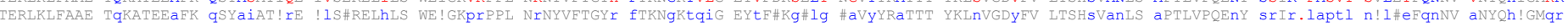
1201

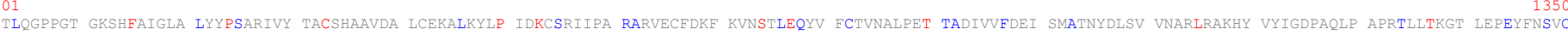

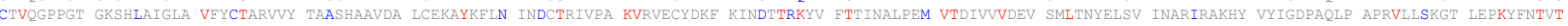
1351

1501

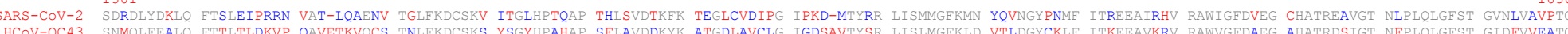

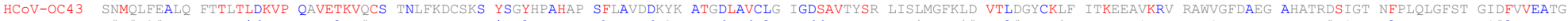

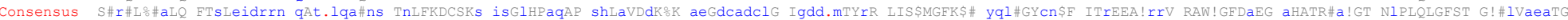
1651

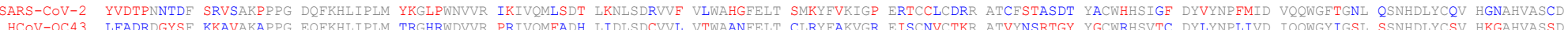

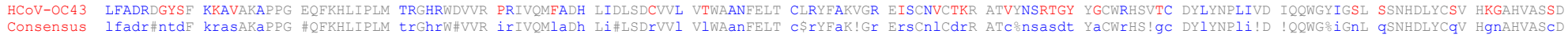
1801

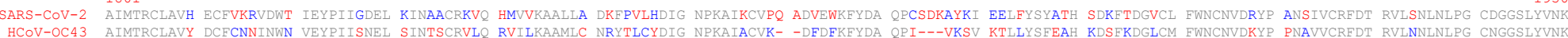

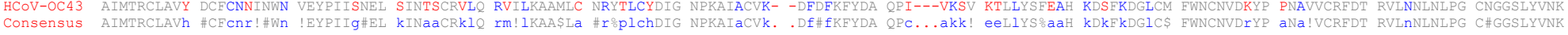
1951 2101 